Sisit of Pants, coll by

(26)

D. G.D. Laviland, Bornes. recd. Aug. 1892.

- Ethaerothalamus insegnis

2 Grtaboligys n. spu?

3 "swaveolens

4D Disepalum cuscomaluoss (ani Decoronatum)

5 Goniothalamus sp. neav. G mavrophyllus.

6 "malayanus

y Mitrephora (?)

8 "ne. Me. Maingayi

9 , n.sp.?

10 Eijlopia steñofuctâln.

II Shuurmansiá anguslijstiá

12 Sozonocarpero in.

13 Coblgala venenosa

10) Baconthofhyllum ancolanum

15 op. n.?

16 Graloricylon formasum

is "microphylum.

19 Garcinia sfen rostiata

20 sp. m. ugeniaefolia. L

21 Calophyllum nir. retúsum

22 Talinandra if cyrtopsda and tiechocosma.

23

sp.n.n?

24,

sfe.n

25

japonica 'vas nitida.

26

" var.

27

28

ni. tristyla

ếvensis

29

tristyla.

30 hosuída 
a

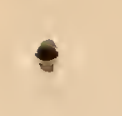

$+$

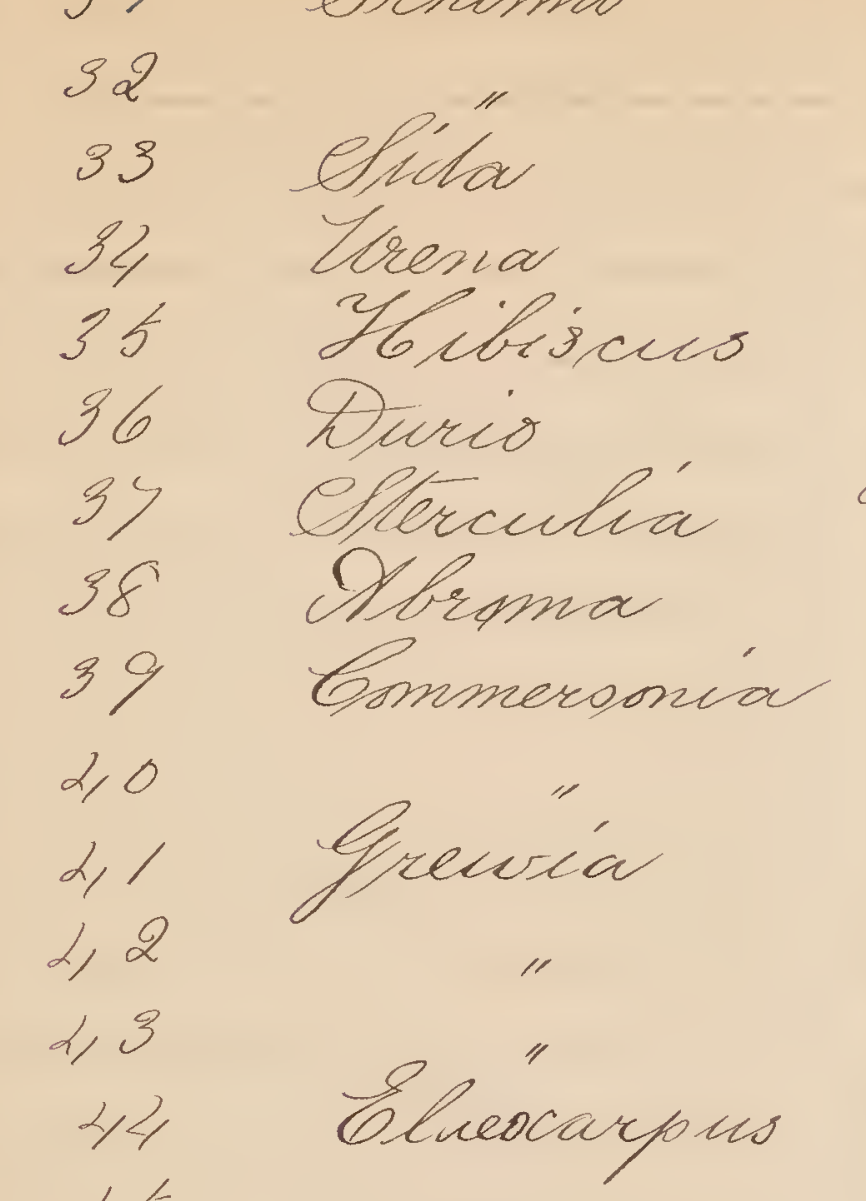

2,5

46

2)

48

49

50

51

52

53

54

55

56

5)

58

59

60

61

62

63

62

Caucheria

Mrevehoa

Gomplicía

outhemió

Santiría

Cfandorcicun

Ghisschilón

Ilex

"

bronymus

viles

\section{Elchima toronha}

chombifléa'

lobala

surratensis

gibelininus?

n. sfe.

angusta.

plalyph hylla

scabrida

ossea = Beco. globulifera

3184.

stipularis

acmocarpus

oblongus

parviflous

pedincularis

n. sfe.

Garambola

sumatrana

n. sfe.

nr. Bilosa

Becracié

n. sfu.

boineense

eryethracarefus.

cymosar

nest.

n. sh.

ni. vagano.

an geniculatá. 
Dracontomelum

tamponoperma

sryloestie

gracules

gen. nov.?

alatá

n.s.

Folaca

Milletia

Desmodium

Mbrus

Eryethrina

Sucuna

Deru's

Songamia Anorarpus

Caesalfunia

Cassia

Peafunia

sercea

mollis

griantéa sp.

mi. A vestita

umbellalum

polycaresum

indica

uleginosa

.3) n. sp.

glabia f. huberula

edules

luga

Borduc.

alata.

up. n. nr, glauca.

fevruginea.

spon nu. bedenlata

n.sp.

sccandens

stipuculat. 


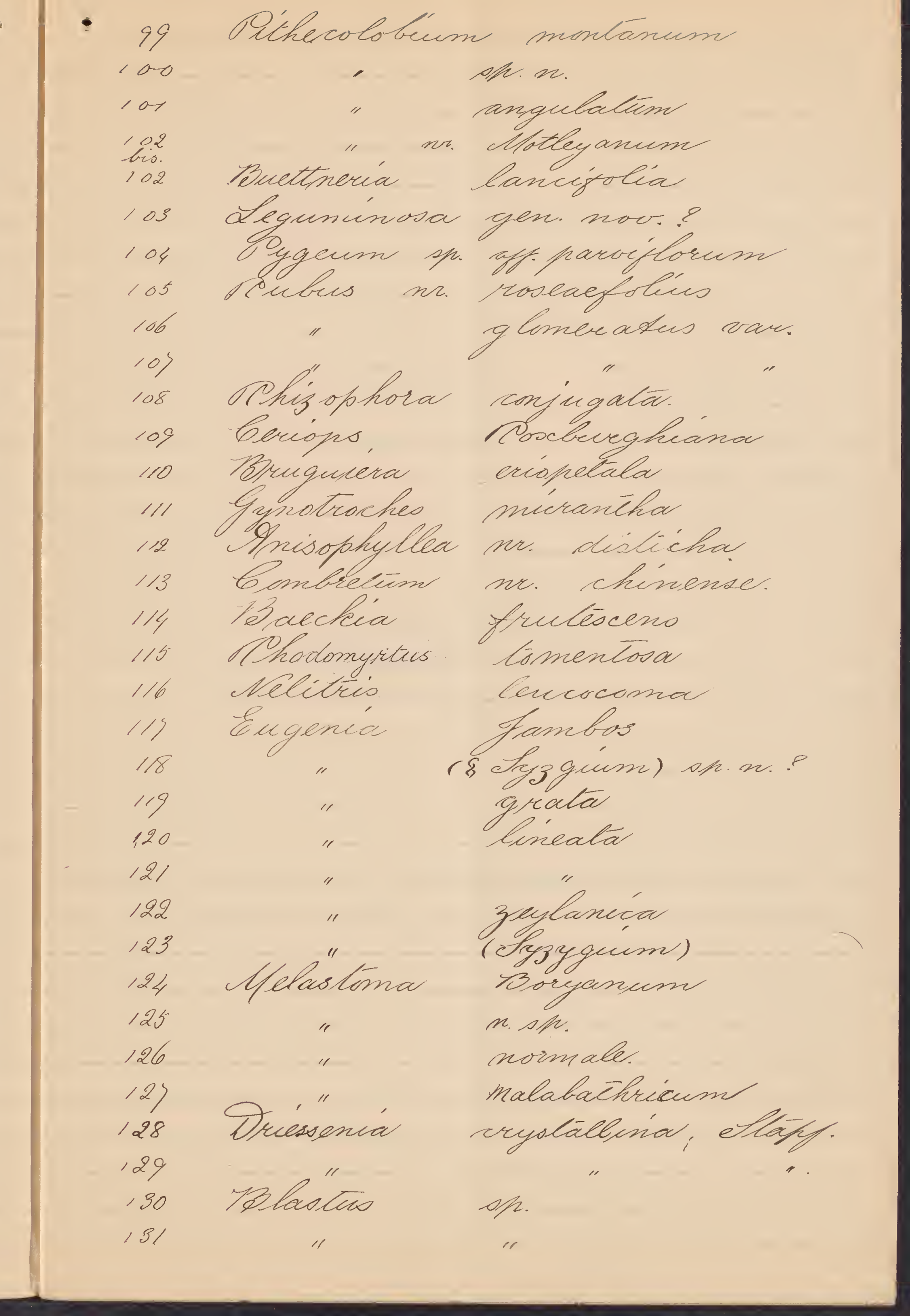


Somatostoma

136

13)

Anerincleistus $O$ Opm

138

199

西

$$
\text { e }
$$

omatostóna

esnevila

1,80

141

12,2

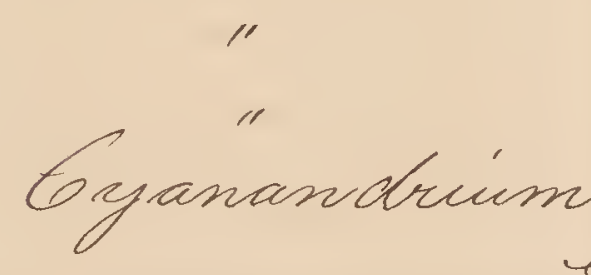

$$
\text { gen }
$$

$12 / 3$

Oomatostoma

134 Dialenia

14,5 Hiarumía

146

148

148

148

150

151

152

153

152

155

156

15)

158

159

160

161

162

\section{Hederella}

Dissochaela

Diosochaela

i"

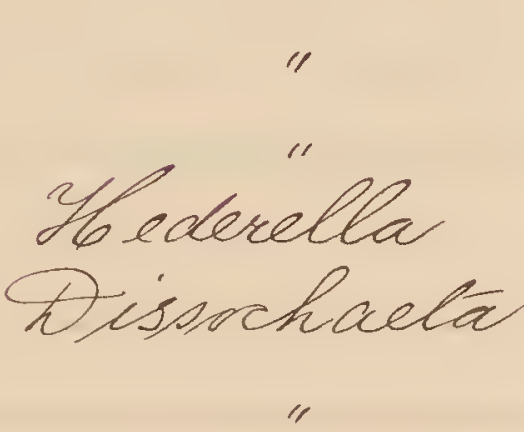

"

Yrmplectivin cliedinilla
Terravir Govis

pance

(a)

roala, brogn

angustif olim secteleifercun,

aniorphyllus,

sertulifercum

eteccariana

borncensís.

lencuifolia

rufum,
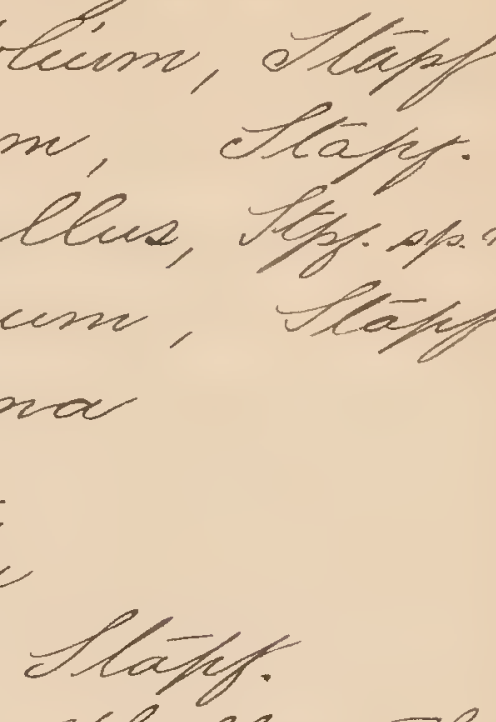
tlatef. tespopin leafey. nov? aff athy llaga 5 merolis. Stee 15.29, phyllagathoides, Matyf. n. sh.

lefirosar pachygyma. stélulata annulares intermedía bracteata

quinulieslinervé multijlora, thafef. Yeccariana n.sfo. ni. Mecrarevand. fallens

petiolare divaricalion crassinervia. muricalá 
191 Getomeles scematínana. 
- 19 Mrithophyllum diversifoluim

195 " n.sp.

196 Mraliacea

19) Viburnum

Gen nov:

198 Mauclea

199 Zncaria

sambucinum

spen. n.

280

207

202

203

Xanthophylim

salerophylla.

ferrea

Mregostémma

Ibedyotís

204

Ophiorrhiza

205

206 Mussarida Afucinaea

205

208

209

210

211

$2 / 2$

$2 / 3$

214

$2 / 5$

216

"

\section{(1)}

vioshyllew

fructóculosum.

ophirenser

n. sh.

of. beco

tene. 94.

frondosa var.

Priónda Db.

Aecananethus

sle. $n$.

Webera

n. sh.

215

$2 / 8$

219

220

221

222

223

224

225

226

227

Anomanchodí

fragrans

Giardenia
clomonicis

$=3425$ ex Haviland. léntarculata

Co,anthicin oscora

Morinda

n.sp. nu. sambosella.

n. sp. wr. hydrangefolea

dedymein

n. in.

rigeda

Neunelliá

sh.

Cephaelis

specusa.

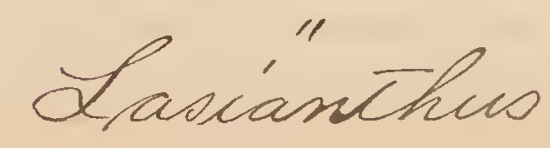

sp.

cuneata. 
Hydnophytum

formicalim - Uubicea

230

231

\section{Vernonia}

Elephantopus

acborea

292

293

234

Milsania

e)soloma

295

Yaccínicim

296

297

238

Leucopogon

Macsa

Pryesine

240

Erdisiá

241

$2<2$

OP "

"

nr.

243

244

Srymplacas

24,5

246

247

24,8

249

250

251

252

253

254

255

254

257

258

269

260

261

elife ax

Linociera

$$
\text { Ghilar }
$$

Ghilocarpus

Dlyoura"

Gerbera

Dijera

ERephanotís

Howa

Fragraea

Gaerínena

Ehreléa

Toumefortiá

Ofromaea

n.s.

. S/a. (G.T.c.e.)

nu. quinalia.

Gelallam

borncensis.

n. sfe.

n.sfe. ni. boncenses.

crassefses

spe.

ofs. n.

sarmentépa.

cumpanulata

cejmosa. 
n. sh esrookia orbiculata 
- 296 Xoranthus

295 buphorbea

298 Berúdeláa

299

Onulland thus

360

Glachiduim

Baccaucea

Antídesma

errugineus

pilulépera

multiflora

is Glochiduín)

nr. Yardneri

liacléala

302

Intedesma

sfe.

304

305

366

$30)$

308

309

360

311

3,2

$3 / 3$

$3 / 1$

$3 / 5$

316

313

$3 / 8$

319

920

321

322

Gelonicim

Crafuicim

Eoccoecaria

ejema

n. s/p.

" ni velulánum

salicijtolicim

"Corchalsic

Pralloties

n. spe.

Slec'deion 1.?

phelifpenais

Heacaranga

n.sp.

megalophylla

sp. wi Tringir.

" velutína

" lopmentosa

"n.sp.

323

Ficis sp

vrientiales

325

hypalenca

caladíf olea

depressa.

vi. spicalüm

n. sp.

n. ap.

326

$32>$

"

328

329

xylophyllav 


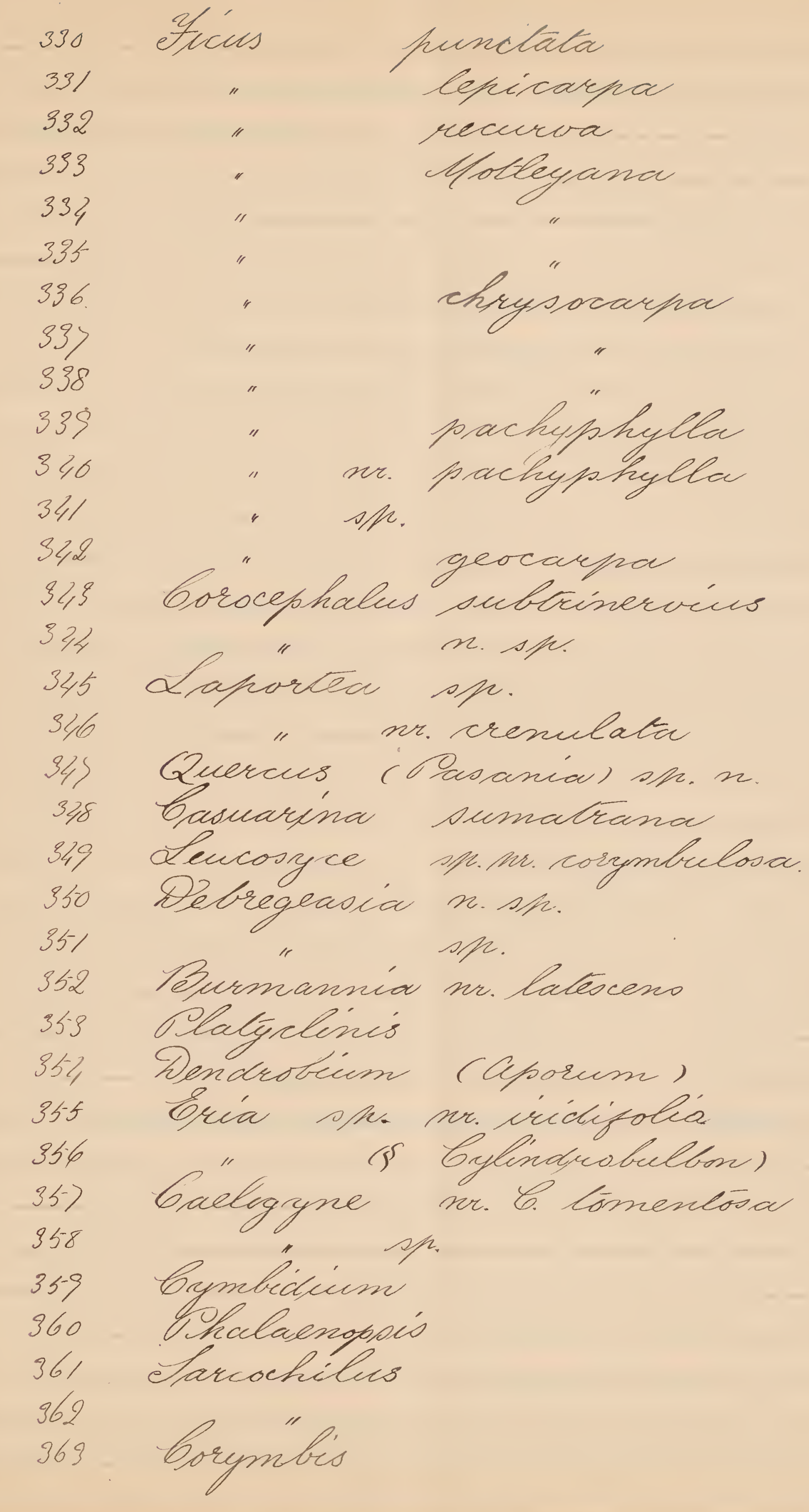


- 364 Goodruera

365 Lfaben ariá

366 labola

969

cho tra

Amoncen

365

$\because 0$

$\because 1$

3,2

고

$\div>4$

$3 \% 5$

$\because 6$

3i)

$3>8$

379

380

387

382

383

(n)

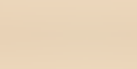

sp.

Elincoune

Ohrynicim

abrosanquinea

seyph hifercon

ne.subulatūn

grandis

sh.

sp. ne. capilation

efr.

Q)ioscorea

Forreatía

bommelína

Dendrocalamuo

finecion

Ordocarnus

Wrichopno

manto

Togonatem

Bacromiá

Gombectoria

epe.

ner pyejétiá

ni. margénata

mudijlora

ofi mo flagellyive.

Yremon

Salciforné
Secma.

macrophy lluss

suferbó

385

386

387

Endotrichum

388

Pacromiticium

389 Nicrancess

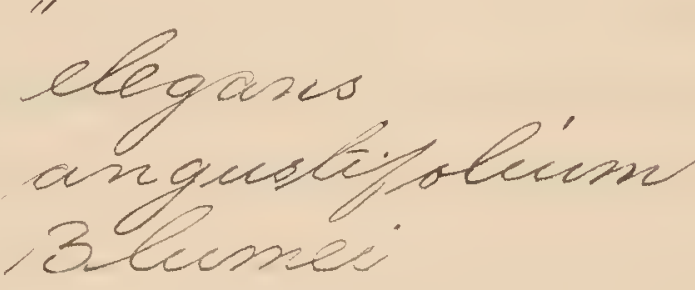

Wo thecs. betiveen chene numbs.

403. Melodorum

404

Gonis'thalam

manubréatum

405

406

Golyallkia

sp. n. ?

exapes

Yseccari

400

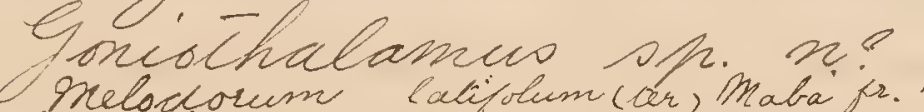

408

Avaria 
Dolyalthia Helodorum Oolyalchia

$4 / 3$

414

415

416

417

418

419

420

421

422

423

(bis)

0,24

4,25

4,26

227

1,28

429

305431 2no If

432

493,4021,5,95: Ho the
2,36
43)
499

$$
\begin{aligned}
& \text { Freycinetia } \\
& \text { Galanno } \\
& \text { Orundinaria }
\end{aligned}
$$

$2,2,0-4,5,00.4 / 200.0$

\section{Kelopia}

ortabotiys Melodorum

twaria

Polyalthía Artabotrys Orly althía helodorum Vevaria

Drepananthus

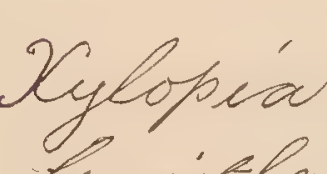

Goncithalamus Solyalchía Al bagnella Lycopodicim

Ireycinetéa ores. (.8) alopecurocies Llabellaba pinufoticion nummulariaefoliciss manibriátion Becrarí 7. Ceyomanní.

=13ecc. $1585,1444$. B.n. (: affi. H. Loweana sh. axycarna fichens aff. Q. purh useare ct. o. oblongar suaveslesis Genteinaí n. sp. $=13 e c 0$. 999. esceloa

(Gyathoralys . m.sp. aff. D. oblecoíolió.

- Beca 653 .

of macrophyllewo

inbicata

sfe.

s. E. Pargarita

1,56

ibs

457

Lacaranga Burmania Macaranga

$$
\begin{aligned}
& \text { af. hypolenca } \\
& \text { Congufolia }
\end{aligned}
$$



- 498

299

500

507

502

504

505

506

$5-0\rangle$

508

509

510

511

5,2

$5 / 3$

$5+4$

$5 / 6$

51

5.18

$=5,9$

520

$5-21$

522

52.3

324

525

526

5.2)

528

529

530

53

532

533
Oolugata

- Securéd aca Of hucuenainséa Gratorytor Elacocarp wo Heibiscus balophyllum Eleavcarpus Gomphía Salaquicum - rymplacos Graloyglon Grevia Sarclaya Hibuacirea

éinomisciem Gluta Thodomartito opugencía Vutis ste. Werciantheo Vitis

Díclea milletia

Mimosa

Thatholobus Ormosia

Derris

Oonganica Bauhiniá

Albiziá $x$ anthophyllum Dalbergiéa cf. venescord.

n. iff. = Bece. $80 \%$ n. op

Pormosacer

Bracolerai.

Éléaceno.

n. ste.

parevizolia

suseclíana

micradesuss

accusuisala

Bolleye

chlorolenca

seliot are.

y. Terghas.

tomescloora sef.

Becrariana (= Esea

(3) var. Ehymsiflora

geniculata

oepcácéa

\section{if. tebea. 6\%.} glabra

procera 
- 538 Oilhercotobuin

535 Revrió

si6 doranthus

59) Careya

$\checkmark 42$ Tomerila sp.

ibios bustorchis

$5: 2,3$ Dienandra

$\left.\begin{array}{l}\text { gert. Szeylanica, Wo C. } \\ \text { agmy paper on Son.) }\end{array}\right\}$

Amather spilicti cio. a. e., Fach.

5414

546

Oernandra

numb. is Memecylon sp. $=2208 \times 1.91$.

Sissochaela

sys!" Dalencia

mulcyllora, Goon. lell...

íracteala, $13 l$.

sh.n. Elettered c.op. B.].

another pp. numb.5:1, but-lestered

2. n... a. is Medinilla longipedunculala.

548 Yhebessia
549 Medinilla coriacea, bagn. microcarfat, Bl.

551

bis.

(Veleroblemma) clerpens, Mtapf. Labioci pachocío

$5+52$ Tachycentría blerodendron

559

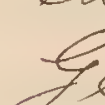
feunsía

554 to

ballicarfa

5.56 Glerodendron

- Becc. y86, zavi 955s. longifoléa $=562$.

559

558 Sydrangea

559

$$
\text { Grodía }
$$

alba.

Commera onia echinata.

561

562 Andraqraphis ballicarpa Vilésc

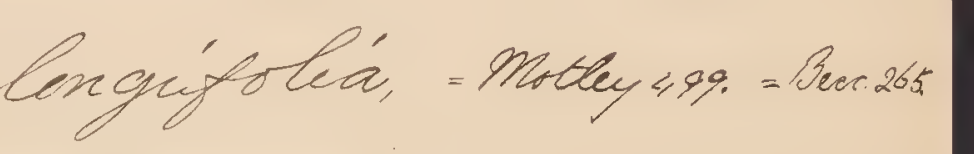
Glerodendron

Vitex Micrechite s/3ec 


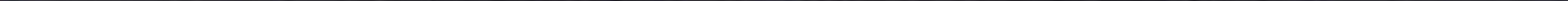




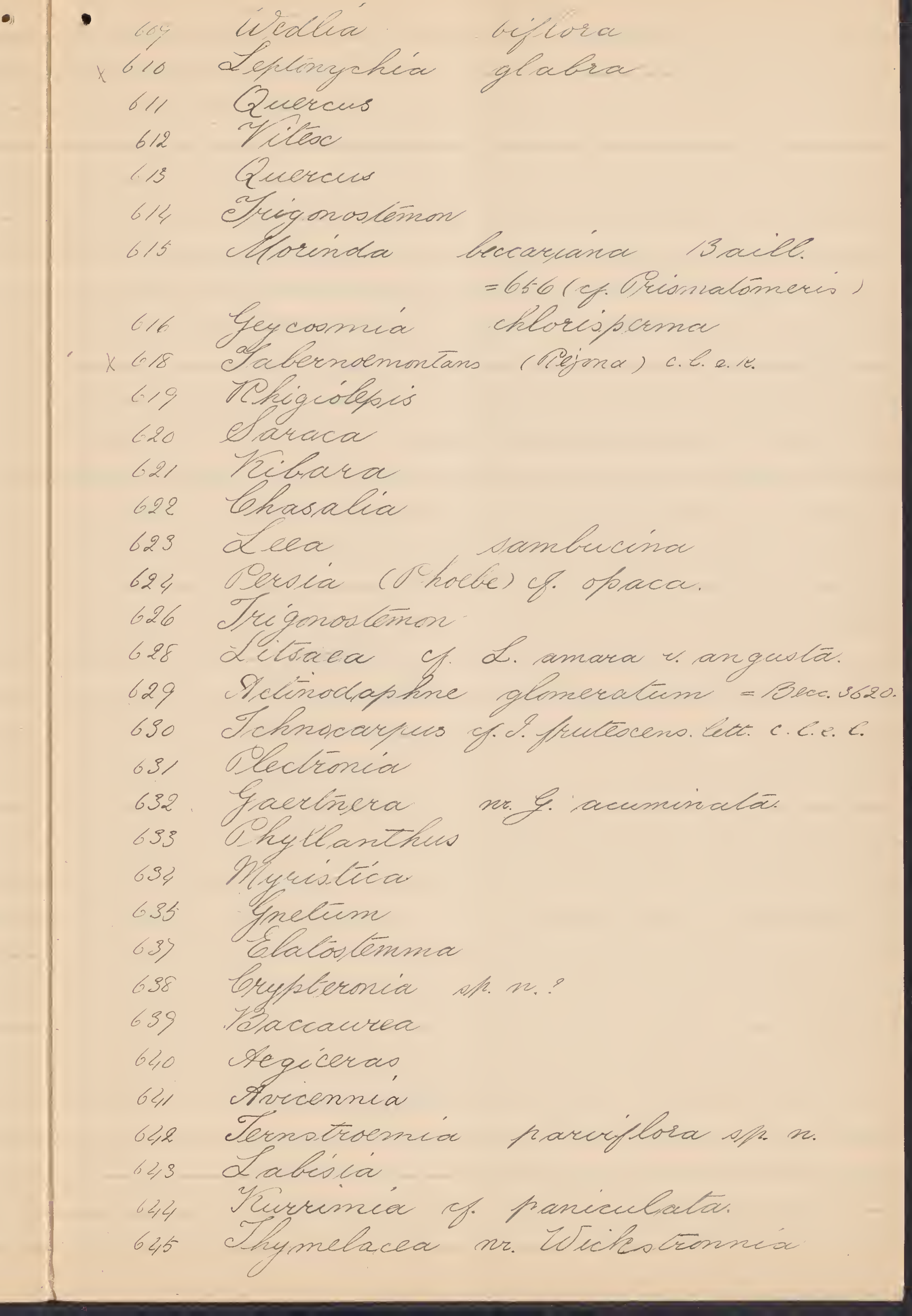




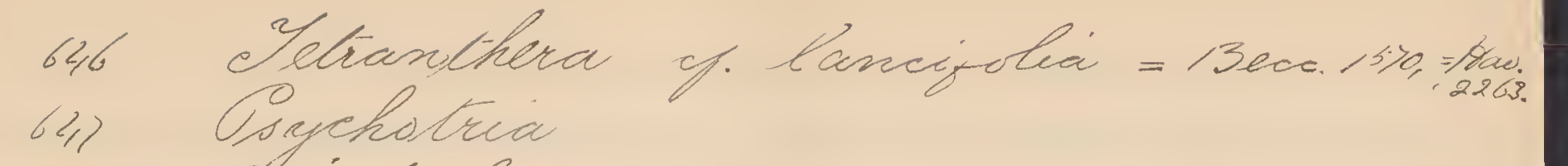
648 


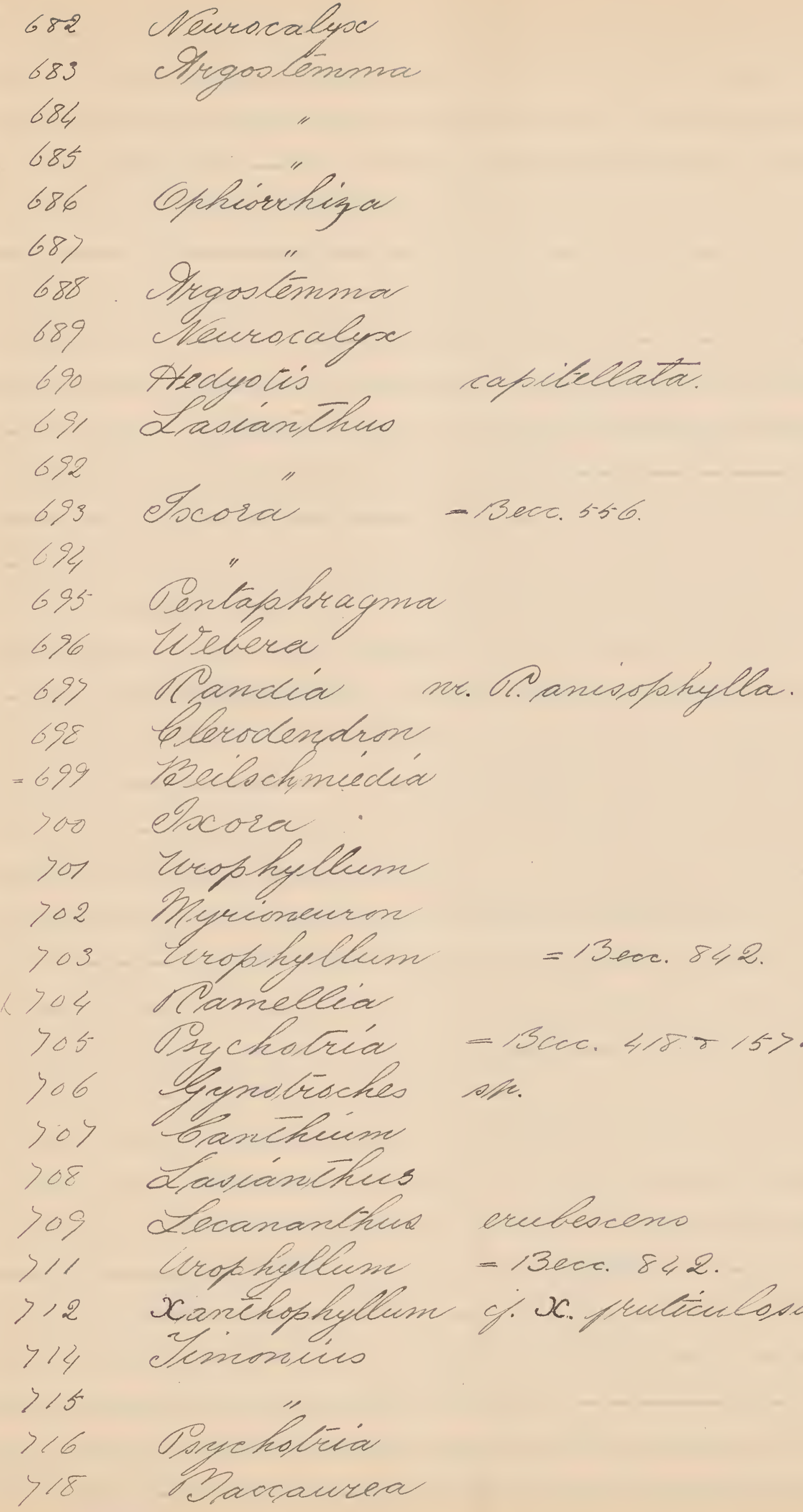

(n)

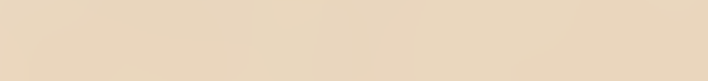


Orvtidesma

Galeariá

Chorosa

723 Oraraleñon

y funticosa.

325

$>26$

$>2>$

728

729

730

$>31$

$>32$

733

734

$>35$

$>36$

$>38$

$>39$

$>\geqslant 0$

$>$ \&1

72,8

344

32,5

726

(bis.)

"Sylócrene

Inticiesma

heacteata

Mecravic

Sporosa

eltêrculiá

Diflýcosiá

Wrophyllum

Dioshyeros

Thedampia

Meleósma

Dicoelia

Campinos herma

claveausá

Yascocarfus

Gracinia

Ilex

Lecuridaca

Litsaca

Mmblyocalyx

Vachisium

Aenslowía

graciliflora

Becrariana

Oanchoni

Hobleyana, císiodes

n. sh. = Bece. $50 \%$. y. castanea

351

$>52$

759 
Yqueum

Glevinitha

Litorea

Ghelscarpus

Ir doveichic

\section{f.}

(b) r.l.)

caliopinglla

759

Geunsia (.)

761

Z i

(9)

Melissma

765

765

766

Villedrunea

Grapharis

Yynaveroné

768

769

bacculus

baccu

As henodesma

Linociera

$7>1$

Crotón

Eurycoma

Maba

$>>5$

) 6

$>>3$

$7>8$

Gomphandra

Lits a

Cyparos

$7>9$

780

781

$>82$

783

784

785

786

$38>$

388

789

790

791

- Iparosa

- yacelnera

Soyehobía

Lunociera

Pabiliónacea

Simplopes

plelcioma

Stsodecá

lleliósma

Jremma

Porychabría

Eurycoma

Siye hotria

$>99$

Splideoma

- Bect. 2959.

L. Lenangiana

- Becc. 3248. af Arinerved apen.

cf. Munsteri

$=13 e c \cdot 9885$

ardisiordes

longifolia

bumingeana

?

wo F. rhinioccrotis

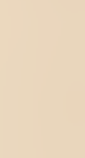

echinocarfia?

of sarmenlisa

longifolía

c. sarmentosa

794 


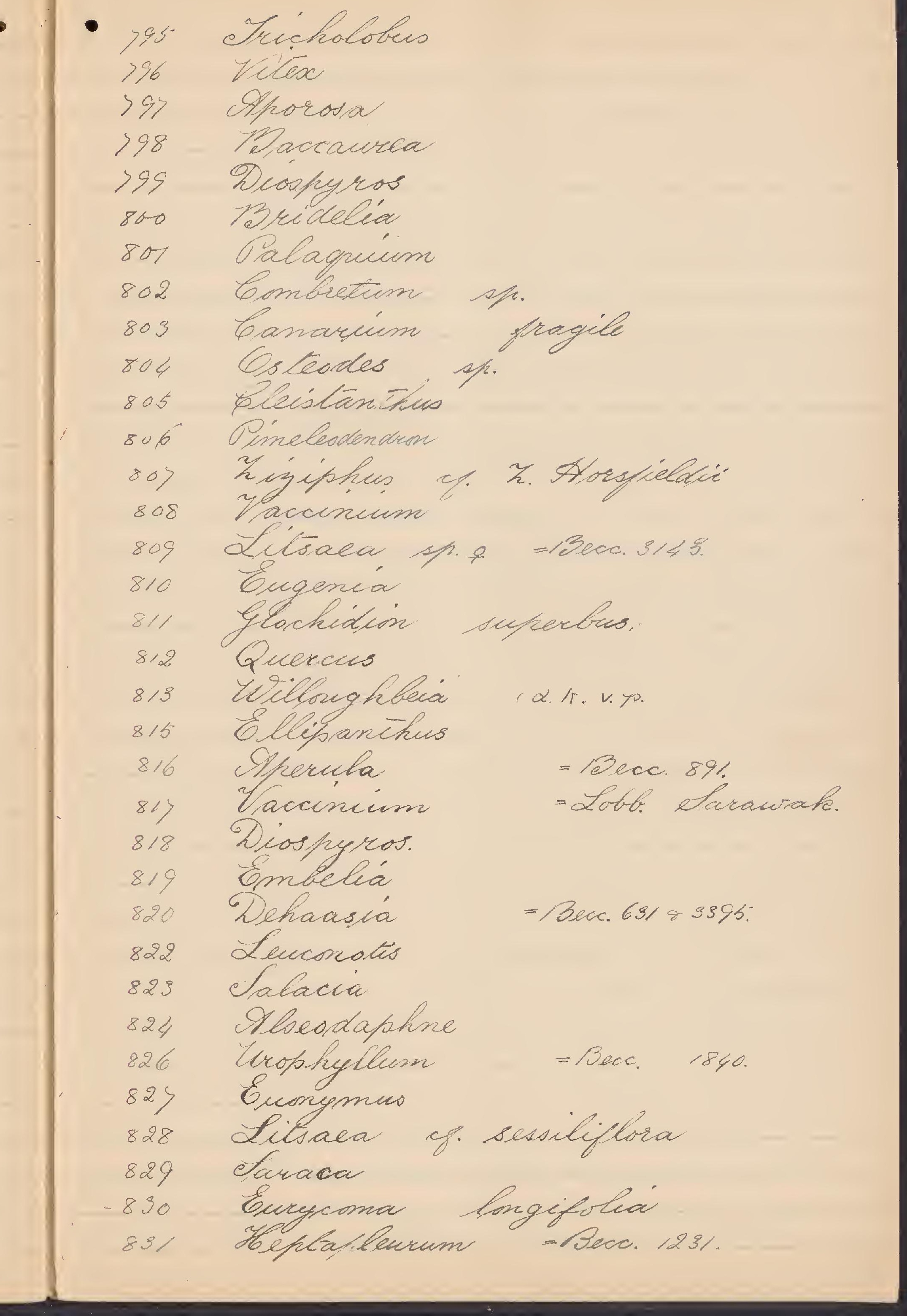




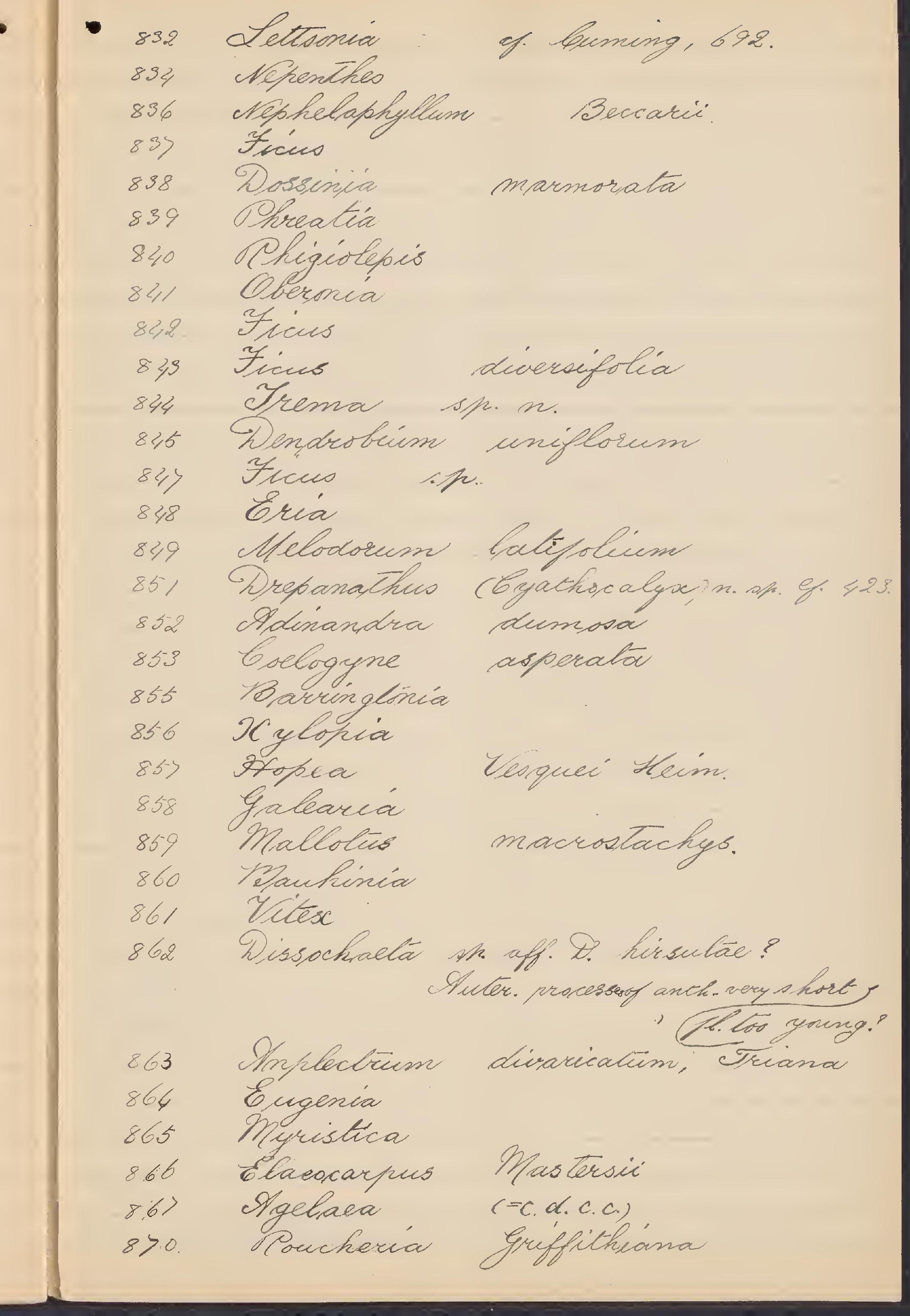


$8>9$ Mcaba

834 Pouchería

875 Trickalobues

896

875

878

$8>9$

bryptocarya Aleladorum

Memecylon

Efaraca

Diospyeros.

Ouonymus

risiphus

Prallotier

882

884

riziphus

885

886

888

Polyalchia Glerodendron

Griflithiana

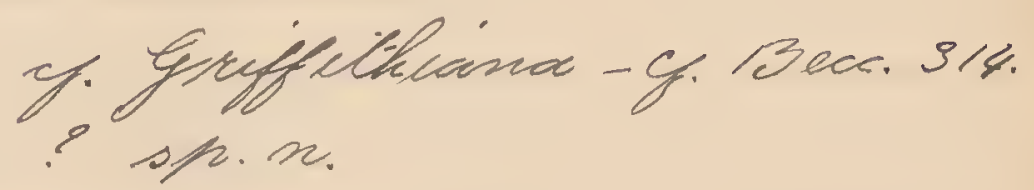
durum, bogn. exdesck.

$$
\text { Tiebera }
$$

889 Geunsía

890 Maesa

891

Dérisanthes

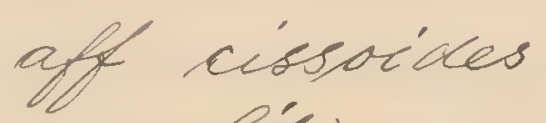

892

893

Buroconelocía polita

894 temor.

E cuclandí



895

elristiania

896

893 Shenodesma Glueor arpus

In acaranga

Dialiun

Hrdisiá

901

Cemenacarpus

902 Teuhanania 


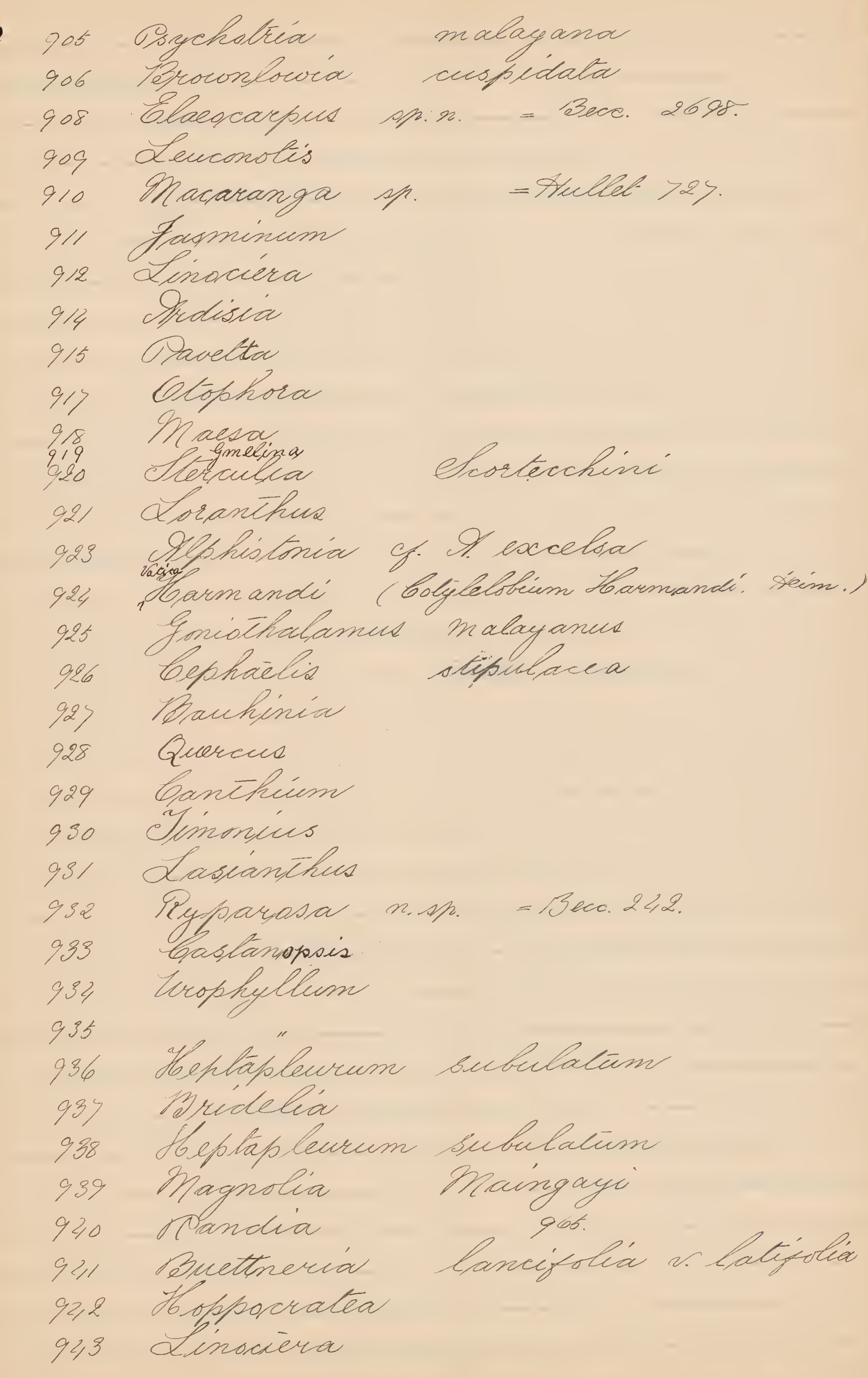




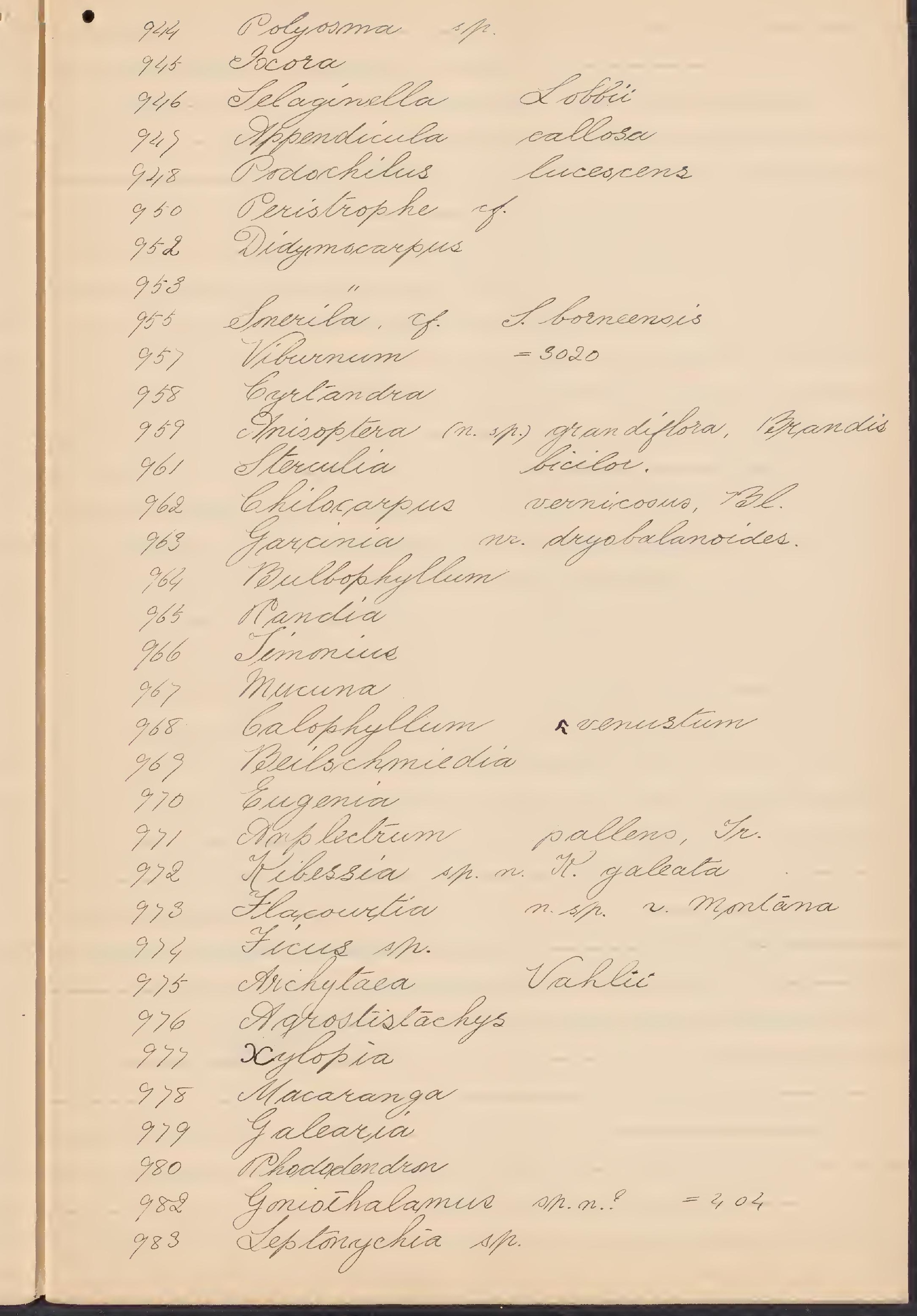




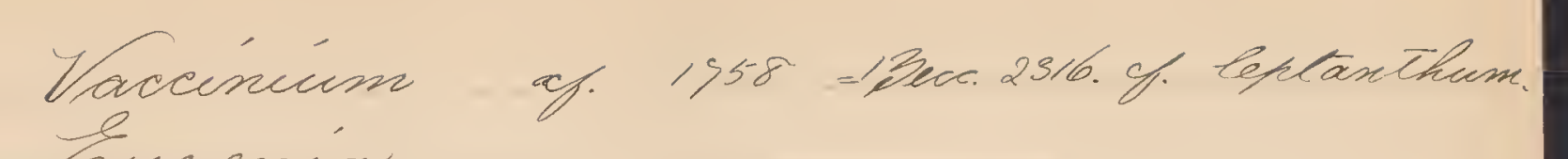

985 bugenia

986 Qvephyllew

98) Allophyllew

989 Hellorigheía

990 Dawhinia

991 Laporleca sp.

of. Tebeck 1530 (a.b.g.c.)

992 Eugenia

994 Pelanochylews

995 equera

99

996

Loranthes

Diospryeas

clelastoña

$=13 e c 0.1248$.

999

1000

Lispsocratia

1001

1002 Somnaropsis Ellepsaneher Pleophyleces

1009

1005

1006

$\cos$

1008

1009

1010

$101 \%$

$1012:$

1013

1014

1015

1016

1013

bis

Xanthop hyllum

Daccaurea

Olyabtia

G unotroches

xyelopiá

Gordonia

Podecca

frarcunéa

- lacocarpus

Y acchinia

ne. crenculata

Gassia sp.

Canavalia

Mrodisia

Allophyllus.

ior Elerodendron

1019 Plablobis

Eomneat very shorlly

preduced at the base, tor enng.?

$$
\begin{aligned}
& \text { glaurar. } \\
& \text { - E. Becrové. }
\end{aligned}
$$
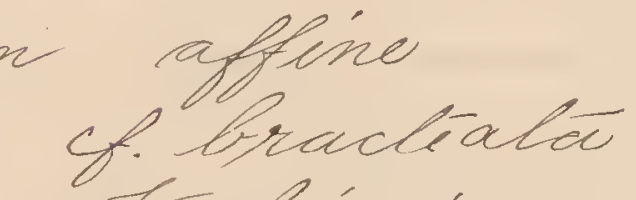

Ten loinsici

- 3 ecc. 1437.

n.sp. ve orandiz

r. dryotalanoides

stepularis 
no20 Gomphia
bis. Gonigiale

Chiqialepia

Hovberi a sevectala.

Buettrería

1022

Dialium

1024

Samplacos

1025

Lucinaea

1026

Ys arronglóniá

Gaertinera

y

1029

1030

faqraea

Iromaea

Yigos Lenma

1031

1082

1093

1036

1039

1038

1039

1021

bis.

104,3

1044

bis.

pavelta

Onomant thodia

Mreriatica

eletranthera

Pemecylon,

Heppocralea

Wíchestromiá

Iremosifinon

Urophyllem

Savraucí

1045

$$
\text { ela }
$$

Elacocarsus

Mickeléa C.'

sh

(1)

beloba

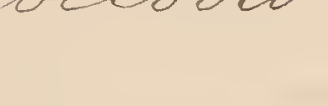

$10 \% 6$ trophyllem

1049

1048 Glerodendron bugenia Sonerila Serotectía

(s oxycentrici) sp.m. $\mathscr{H}_{3 \times 3} !$

1029

(Lpipet. stam. Choter, spwrred!'\}

1050 Aspronía Biemecytor smilacyfled, etracina Tocora 
Potentilla

Hravilandía

enenceana

Euphrancia

1063

Lébtosnermum

cibur

Mysine

Thodadendron

Eureya

Dolegosma

Quencus

1069

$10>0$

Daphniphyllom

$10 \% 1$

10,2

10,3

1054

10,5

10,6

1093

10,8

1079

1080

1081

1082

1083 eliranciaes a

Goprosma

$$
\text { Urophylecom }
$$

Percera

Dichotricheom Hoplouagis

or apeles

doranehus

Mhododenderon

"Nubecs"

Vaccincúm

1084

1085

Gauletheria

1086

Díslycosiá

1089

Eles

1088

Camplacos

1089

ombelé

1090

Plyeociáa

logt

1092

sarvecla

borncerses

lepcopadiódés

fornaveolens

Recuram

levecules

dasyphytha

ericoides

reticulala

Eto ofere.

Ibavelandu"

- barneense

unlegerifotia

Ho oberi

crassicaules

subanucuen

clepressa.

asperifotcum

mickanthar

ercioides

sabaensis

busecfoteim

docoic

arvi

beoscifotien

borneensú

zenabaluens

revolula

Bisupterder

upiracacódé

capiléllata

- iarancóa

hyposhnyllar. 
Podacarpus

1094

1096

1098

1100

1107

1102

1109

1105

1106

1105

1108

1109

1110

\%II

$11 / 2$

11,3

114

115

1116

11

77

1118

1119

1,20

1,21

1122

1123

1124

1255

12)

128

1181

1132
Eugenia

braelogryne

Bulbophyllum

Mdinandra

Gastanopsis

Tolyesma

Simplacos

Lindera

Yaccinium

Loranthud

a orantho

Gugenia

Micuobropis

biaeocarpus

Eugenia

Embelía

Ilesc

Gastanopses

Ssychotría

Combelia

Oigeum

Lasianthuo

Hedyatis

Heclyotiós

torophyllem

Sletrís

Chéma

Litsaca

Byrtandra
Gaccinciem neriéfolia

cupressina

ampullaria

papillosa

coriaceum

cerrucosa

turebinala

baadeosum

defusa

calsia

sabaenois

Deyrlillaw

ram ijtora

Sericeus

tinaboluensis

torluosal

subencrois vacainecagolia var.

densyfolia

minulifolea

vocarficum

ecunecreco

mackostegéa.) terophydlum longidens meacrostégúa

fuchella

cybhandrum

Jolis looa.

requela

Bevijolia

ceylanica

Slairser

coriaceum 
Thodadendron Vaccínéum Lasianthus

Lefitoopermum Mngrtus 
166 lécalanophoia

168 PMemecylon

1169 - Ple Ehiandra

1190

113

1152

1193

1179

1195

1176

1198

1178

1179

1180

$4 / 81$

1182

1183

1184

1185

1186

1187

1188

1189

1190

1191

1192

1,93

1194

1195

196

1.99

1198

1199
Amfiledrum

Bedinilla

Ohyllagathis

a onerila

hiciesseniá

Dicimys

$$
\text { " }
$$

$$
\text { " }
$$

Gattersonía

Thododendron

ciplyposiá

Dacryolicim

Sleysecí

Clex

etymplacos Begmía segonía

Maesa

Goodiá

Mecbes

Nubus

'Sérculiá

Heliciáa

OChers

esistania clon qata

lancealalün

Hooberi, Itafy.

homac andrum

stephanoslegiá

uniflora

graciescula

glandulegera

siberita

"

boncensis

cunecifolium

rufa chrysothrix elalüm

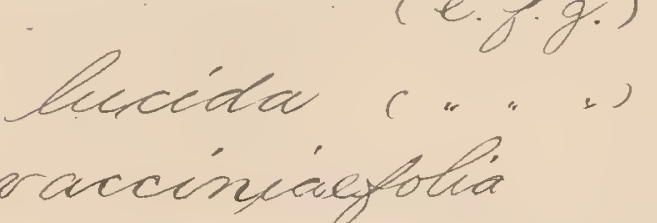

var camplotoba aden ophylla Bécubidacé

borneensis inosleḡé

indica

subunifolioalá glameralus

rasagfolius

translucene

erratíca

borncenois

bilocularis 
etonerila Olesc Diplyecosiá Embeliá

Grírcaulon he sranchus Onloranthus Elatastemma Gromphandra Diplyrcosia Gacetinera Mrdeupia

yécus

$$
\text { " }
$$

Goniothalamus - Labía

Elalosténma

Oeferomía

Liscaea

Taportéa

bryetiandra

Inedinilla

imilase

Penláphragma Ohlomis

Eernatioemia

Qlea

Laportea

terophyllew 'Tavianía

Alpinia

ctolanum

ofonfraliéns sulchella

sficiata

punclielala

fhacadincía

Hooteríanum

brachyolachyus

lichoncuram

lyoipetala

memecyloxides

rufinervi's

amabilis

oxyphylla

$$
\text { seliflorus }
$$

Molleyama

Iinabaluensis

stenopelálus

harviflora

thalictioides

candida

caulijtora

stímulans

Burbidgei

lasioclaclos

reucophylla

surantiaca

rugosa,

Lowé

pterdcaulió

stimulans

salicifoslium

actinidifolia

biflorum

platapselála 


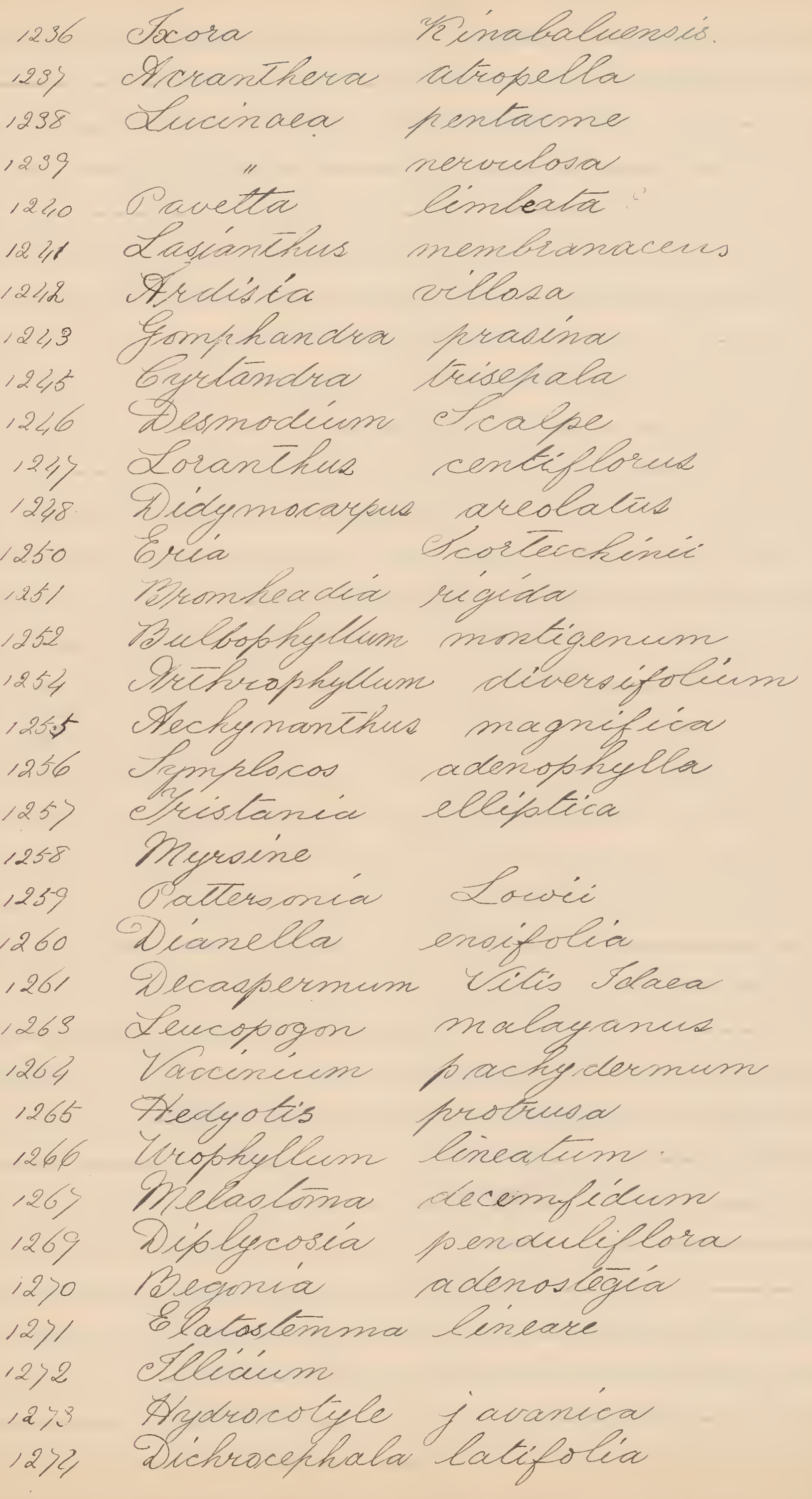


serpena

Teccarcanum

wrophylla

microchrix

Gogniauscii

cordalus

Tinabaluenois

tenuifotía

maculala

borneensid

Ohyllagathis

Dissochacta

elliplicia

hísula

\section{Dichotrichum}

Scaevola

efrathoglotis

Gryitandra

Smilax

Ghasalia

Mhododendron Vaccincum Diplycosiá Mhododendron Lasianthus Gremna

Mrendicula Mibessiá Glochudion Mnaesa

Gremna Lasian Thus Glerodendron Begenía Srattia bractéalúm

Tronsigí

auria

trisefrala

adoralissima

gracilis

lacteum

micrantherum

hinifolia

durionifoliém

membranaceus

cauliflora

congestá

tesselala

ienuistýlum

indica

cauliflora

Kinabaluensis

Yinabaluesse

oflongij olía

borneens ís 


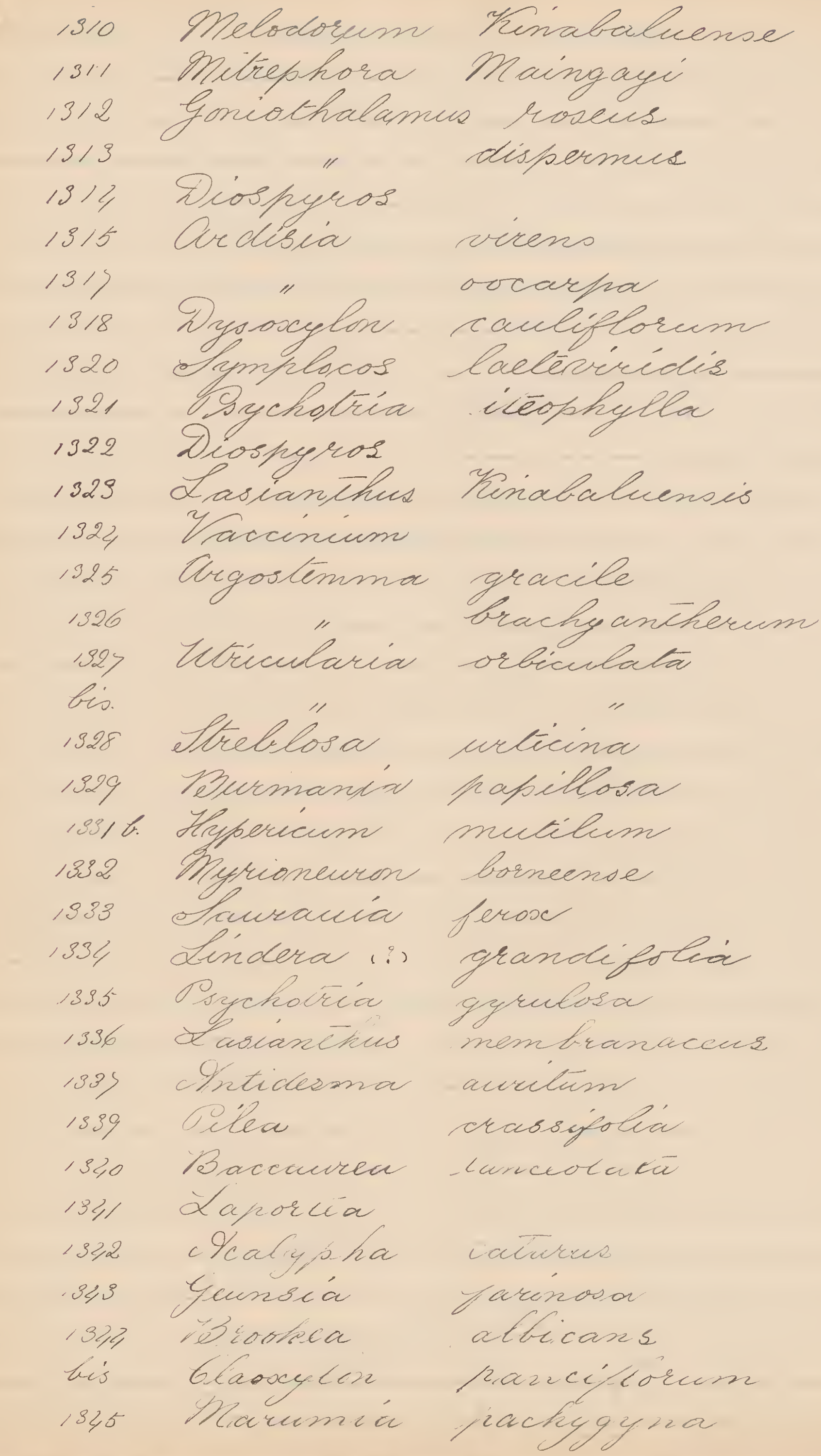




\begin{tabular}{|c|c|c|}
\hline 1346 & - Mamvolfía & - verbentína \\
\hline $\begin{array}{l}1347 \\
3 \geqslant 8\end{array}$ & $\begin{array}{l}\text { becuoseyton } \\
\text { Kevionietta }\end{array}$ & $\begin{array}{l}\text { jiancý lozum } \\
\text { suciunicosa }\end{array}$ \\
\hline 1349 & - qula & wenenosa \\
\hline 1350 & Oolyganum & Gnuivense \\
\hline 1354 & doranenus & estéfitalies \\
\hline 1352 & dd leya & ascatéca \\
\hline $195: 3$ & $m$ & debile. \\
\hline 1354 & mere & rhomboidea \\
\hline 1355 & Pussaenda & molosad \\
\hline 1356 & $"$ & coccunear \\
\hline 1957 & $"$ & \\
\hline 1358 & 6, ampianumota & - celebica \\
\hline 1359 & ymera & lejera cely G \\
\hline 1960 & & \\
\hline 1961 & $c^{2}$ & zocna \\
\hline 1962 & Ehes & cnabaluensis \\
\hline 1363 & & bipula \\
\hline 1364 & sa & têris \\
\hline $1365^{\circ}$ & decuconotis, & anceps \\
\hline 1366 & Wendlandica & funuculalá \\
\hline 1367 & Glethra & carescens \\
\hline 1968 & ellea & acrophylla \\
\hline 1969 & the colo & a bigemincusm. \\
\hline 1370 & clacia & crifor \\
\hline 13,2 & sisecs & - hastála \\
\hline 1373 & clidinandra & escelsa \\
\hline 1374 & Anlidesma & Ploritsü \\
\hline 1375 & chroa & \\
\hline $\begin{array}{l}1336 \\
\text { bis. }\end{array}$ & Grodia & \\
\hline $13 y 3$ & loslégeá & ensecs \\
\hline 1399 & arcenia & Garalandii \\
\hline 1380 & Gandía & densiflora. $=16,6$ ex Yaviland \\
\hline
\end{tabular}


Bachionia

esccurrens.

1984 - Mremecyler

Disisochacla

laevigation

annuluta

1386 Cratoscylon

bis Protiscá

holyasitfum

brachy the

1387 Etchizostâchum Blumer

1388 Gasearia leucolepis

1989 Berotettia

Suchatria

Deniachloa

1390

1391

1392

1993

Bilea

Elalostemma

Gares

alpeation

1395

etchoenus

malayana

Fohicina

bulbainriśc

1396

1395

1398

1399

etciépus

rara

14,00

1407

14,02

Migrastis

apogon

Deschampoía

Deyeusiá

b arex

14,03

iloc

1405

11,06

1407

1208

"

Sladuin

inundatus

Elarber

canina

- Slescuosa

efilenca

filicina

hyteophyta

fusif ormís

samoinse

¿Choenus

Torache

melanostachyus

1409

Colismenus

14,10

aycopodicim

teunthiama

compooitus

cejlanicum

Eelazo

teightiánusn

1402

clavaliom

14,09

1404

12,05

Selaginella

casuarenored

cautésens 


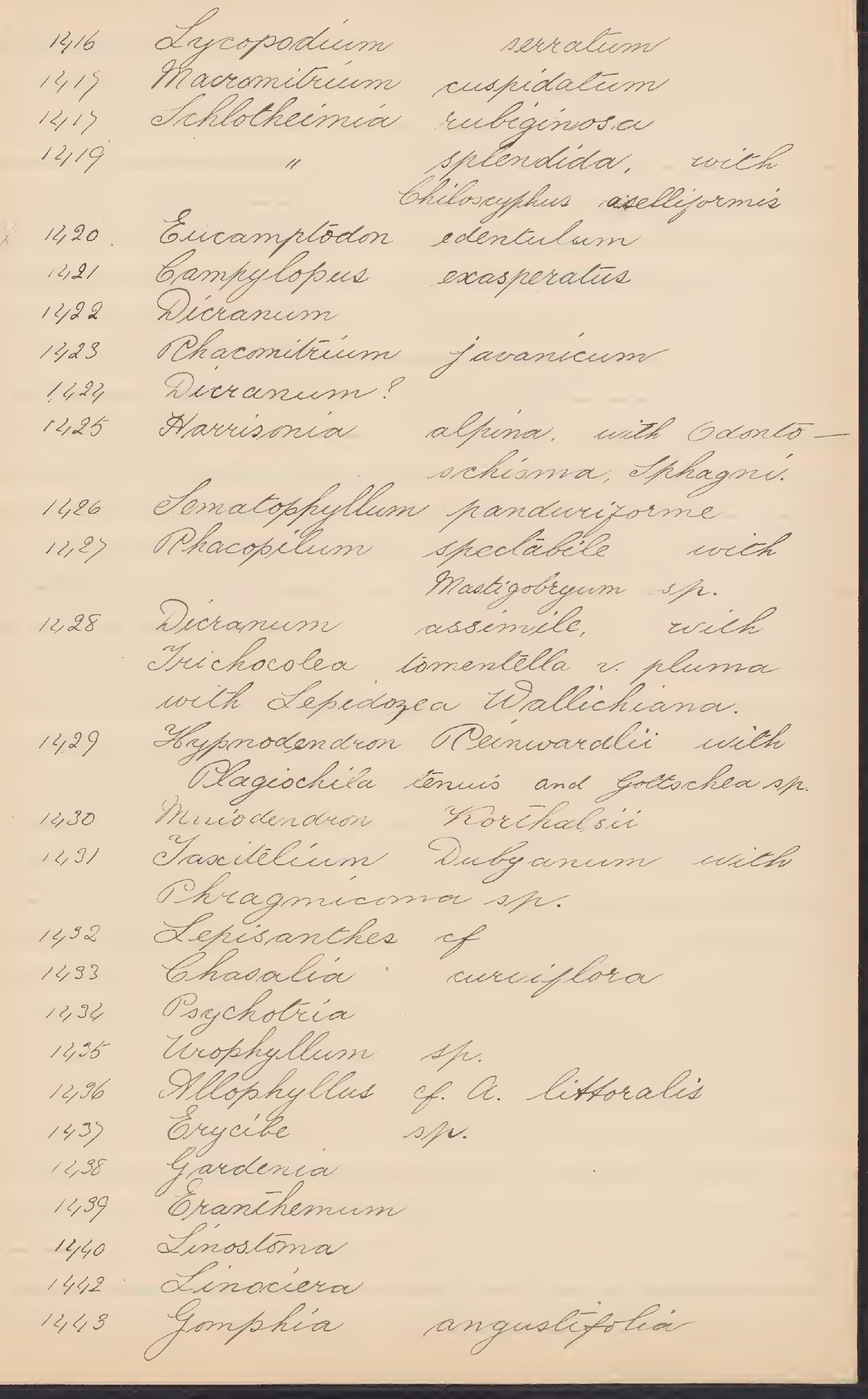


Dalterqia

Cmphalea

Candía

Tomonuis

Guettarda

etéderocyelon

cumosiá

écomonies

Gonnarus

Drosera

Longéfira

spe

1450

1451

14,50

12,53

12,54

14,55

1457

1458

14,59

460

1461

14,62

1469

14,64

1465

1466

1463

1468

1170

14) 1

14,2

14) 3

$14 \geqslant 9$

14,35

14,96

-43)

1438

Gasuarina

ejóvonius

e/abernamontana

Mododendron.

$-1849$

Boca

Machycentiéa

Ohiqiolefis

Eralosylon

Gleopma

Druction.

Borínda

Provenclocurá

b) fichandrone

Wolchandrone

terophulluse

Gicuría

Elacodendrox

Cauchería

Acrostichum

Aoplenciom

Angeóblews

Cisplencion.

elechomanes
Lettsonía

sfeccosa

of. Bareanum

odoralue

e.re (Mgiana)

Sh. CHaviland souss $=114$, butilis not ily. of sour set.

Lormaduse

anguslif otcion

Becrarí

I headie

leploptiylla

subiratundum

Griffeithána

costülatum

Aiplazium) biscricule.

evectar

borneense

microchilum 


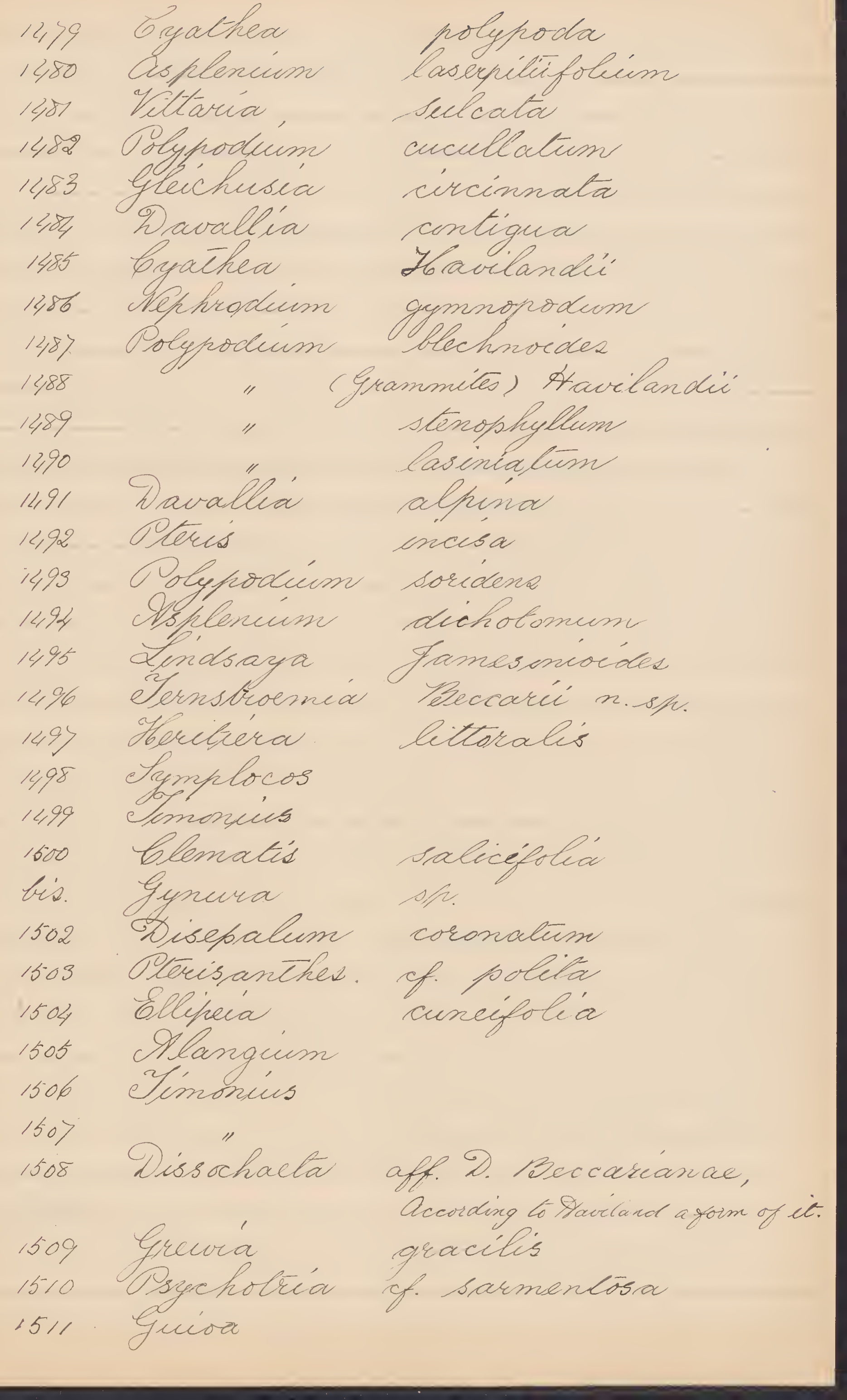


1596 Prallotur
1595 Gomarue

1598 Gedysanchera

1599

1600 Leplonerehó

an Taramería olop hora

1602 Oinheroloticim

1604 Nowrea

1606 ifsorasa

$160 \%$

1608

$16>0$

1612

$16 / 3$

1614

1615

$16 / 6$

1613

1618

Lindentergéa

Lepidagaleis

Gyrinopsis

aff. L. parviflora.

claces s/e

Srgostemma

Baccavera

Quercus

Xanthophyllawn

Wilkecolobuim

tuhec'́osic

1619

Elacocarpus Ilulleti:

1620

Xanthof hyllum

1621 - terculiá

1622

Euquencía

1623

1624

efromelocos

Lepisanetres

Nhiquolepis

1625

1626

¿talacía

1627

esaciaurea

1629

Brabobres

$s / 2 .=2958$

630

Mnemecylor

Yitex

1631

ellênonurus

Glaeacarpus

Fora

$s / 2$

- Becc. 3.580

laev's

1632

1633 
- Cancirá

1697 Elaevcarfue

1638 Dibterocarfus

1639 elicownetocicá

1640 Leritiéra

1641

beo.

Deoapyros

y accaurea

$16<2$

cramplocos

cerres

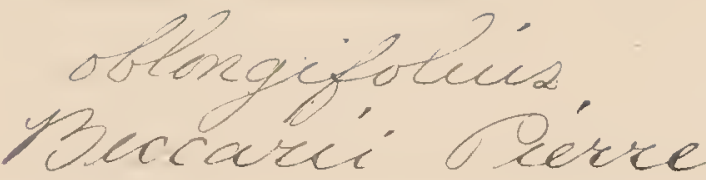

$16<3$

1642

16 es

$$
\text { yices }
$$

$16 \geqslant 6$

buchanamiá

i6e3

1649

Peschymanthux

1660

occónanetheo

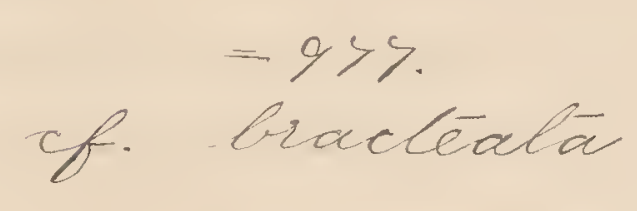

1661

Dryuítica

$=1624$

1662 Drdes

1653

Visepralume

Hompelobsis

1654

éacicerea

1655

Sourea

1656

Cusnonies.

1653

(

1658 Goffea

rectucléa

1669

Buchananca

1660

ciéca

166

Goffea

crophelluna

1662

1068

Ancioptrytla,

1666

écurmannca

1666

Taronica

1663

Dise fudia

1668

Aasianthus radicans $=$ Pottey $>b$.
multiflora

$=1892$.

anonalcens

geraceles

cf. Fracteata

1669

Gasearia

$16>0$

Oachynowarpuo invbonalus 


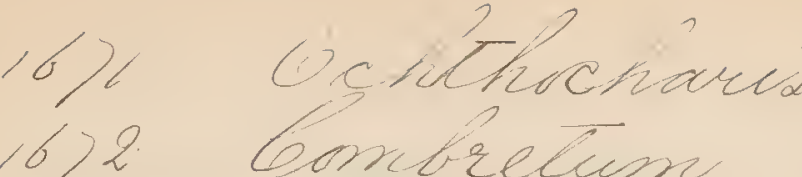

1673 eforea

$-1963$

1674 Pentace

1676

1676

dasuanteno

Loranchus

$16 y$

Fctinodaphne

$=15 y y=\sec .910$.

1698

Ellopanthus

1679

symplocos

1680

1682

1683

1684

Orly alinéa

clecinilla

Grewa

Eugenía

1686

1686

1689

1688

1689

Grevía

bonocephalua

Garcinca.

¿ fauraucó

Mlstañía

1690

1691

1692

Ocumellía

Yibraurea

Yebera

macroplera

1699

tamadéra

sumatrama

rileterablemma)

gracilis

1694

Goelospernecurn

\section{Califítia}

vecblisuckeces

Desrocisseauscí

n.s. = Becrari, $1>82$.

1695 SPurea

1696 Garción

bis.

Pulinga

1698

1698

1699

Galophylu

Eugencía

3raccanrea

dialicim

$1>00$

Quercus

1302

Afrercha

1)

Golumbiá

If

$=212$.

indéa

yot

=Beca. 89\%.-

serratijo liá 


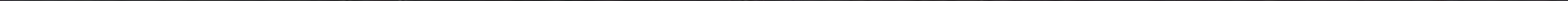





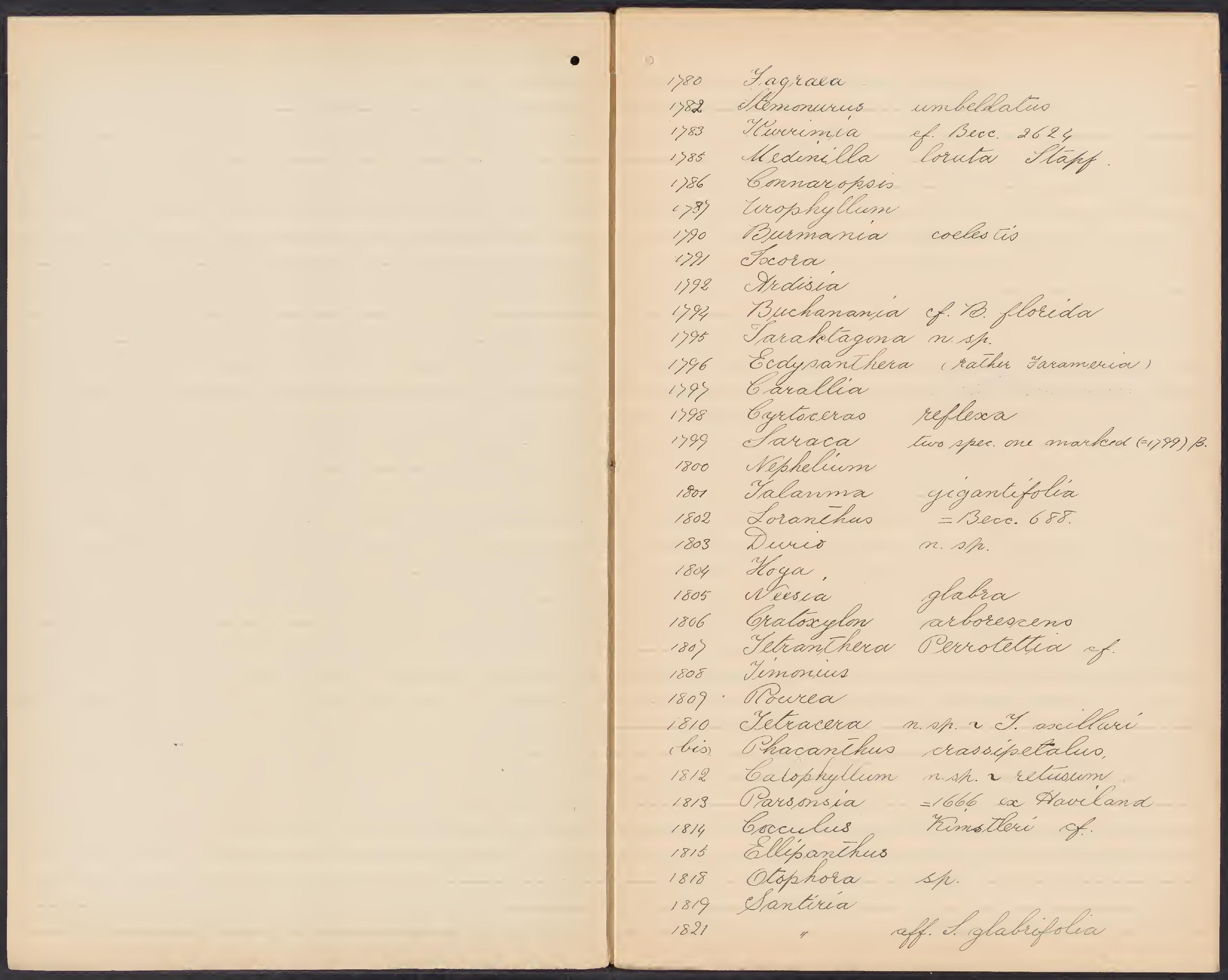


1823 eleyplophora

1824 Gofocura g. heila

1825

etaraca

1827 Clantiría

1828 Ebermaiera

1829 bis.

1830 i.

1831

Socola

Socymilie Eugenva

$=16>3$.

1832

1833

1832,

1835

Tayea

Bemecyton

1835 Delaquum

1806 etetramerisla

189)

terophyllum telrooleca

1839 Agelaca

1840

1841 baloph um telerodendron

1842 Melopiáa

1843

1849 Gevrimia

1845

1846

Oporosa

$184)$

1848 Melodorum Grotón

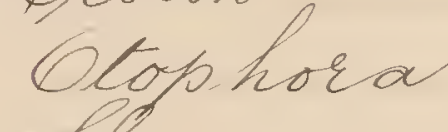

1849

1850

Artabobys

1851

Tromaca

Meris anthes

1852

Golumbia

$185 \%$

Barrinqlónía

1858

Buidelía

2 rugosia

$-145>$

gracilis, A. suaveoleno. denticulata serratifolia 


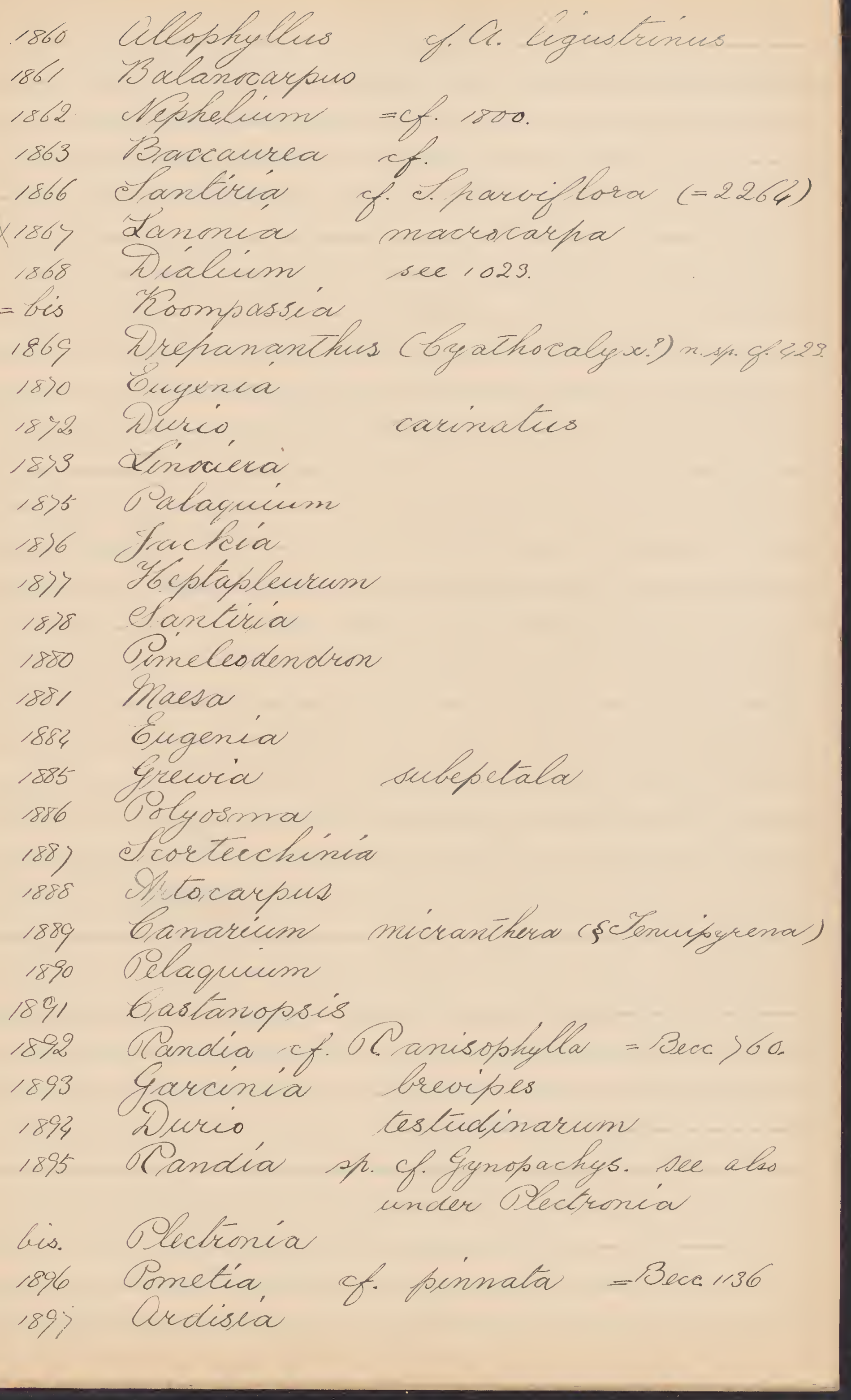




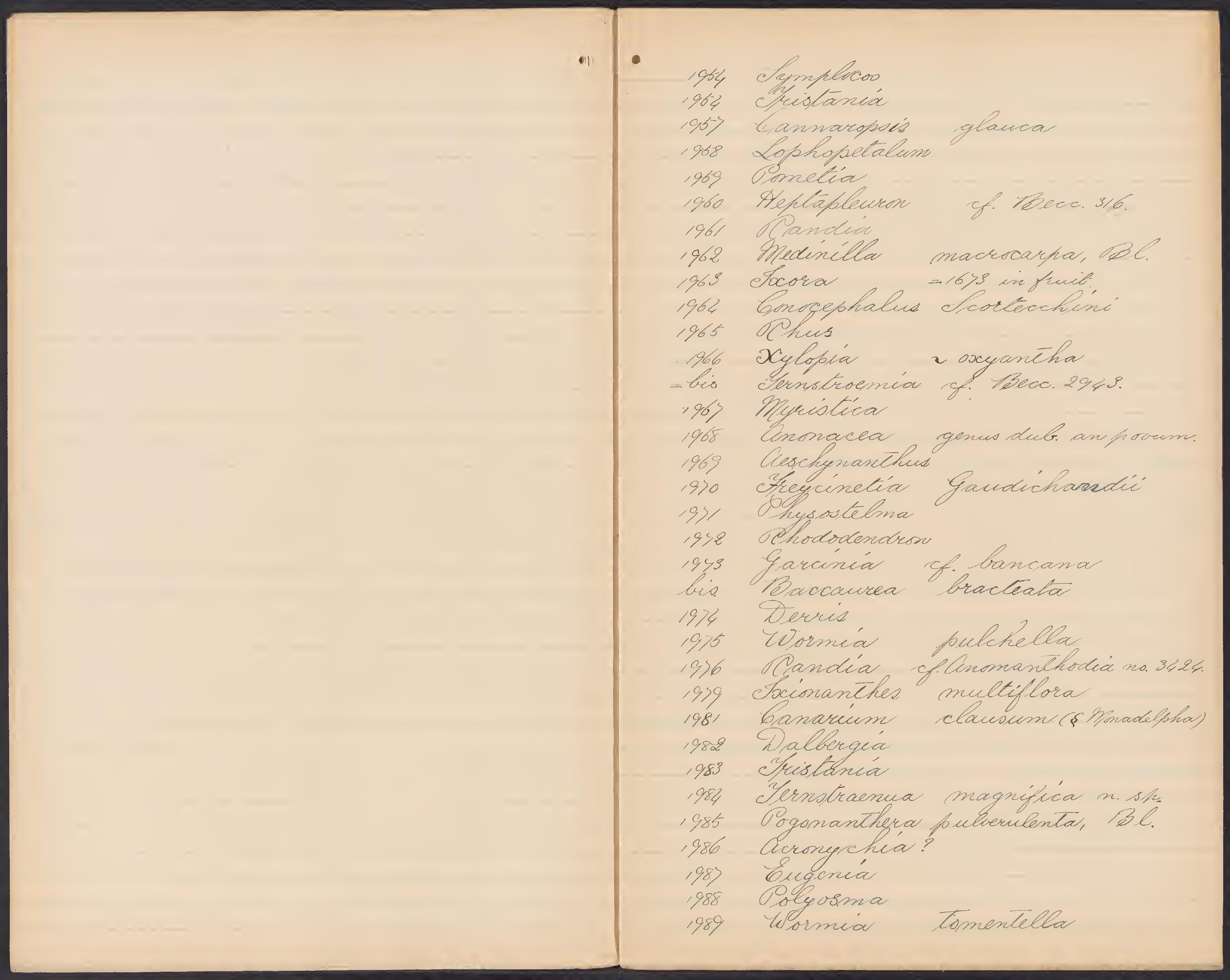




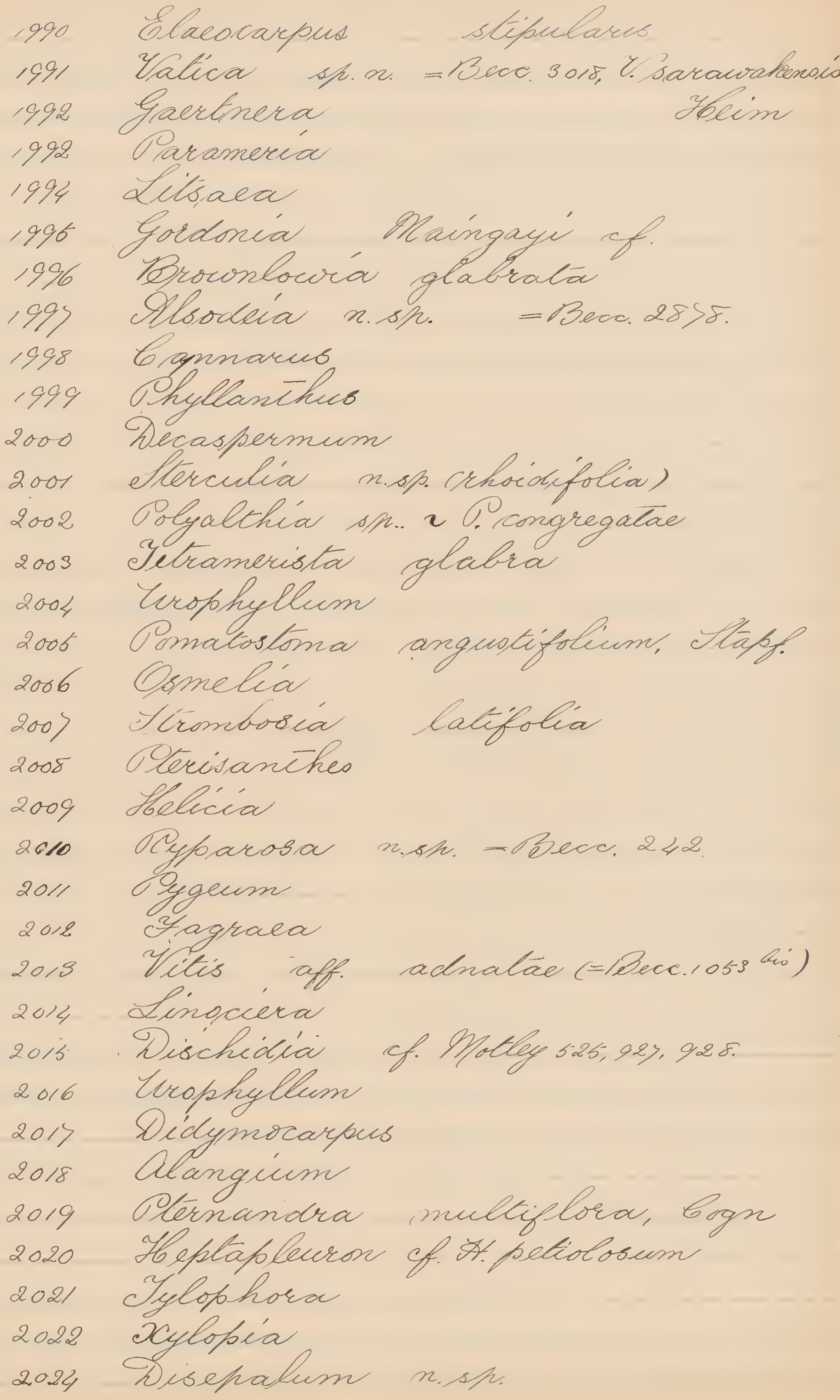




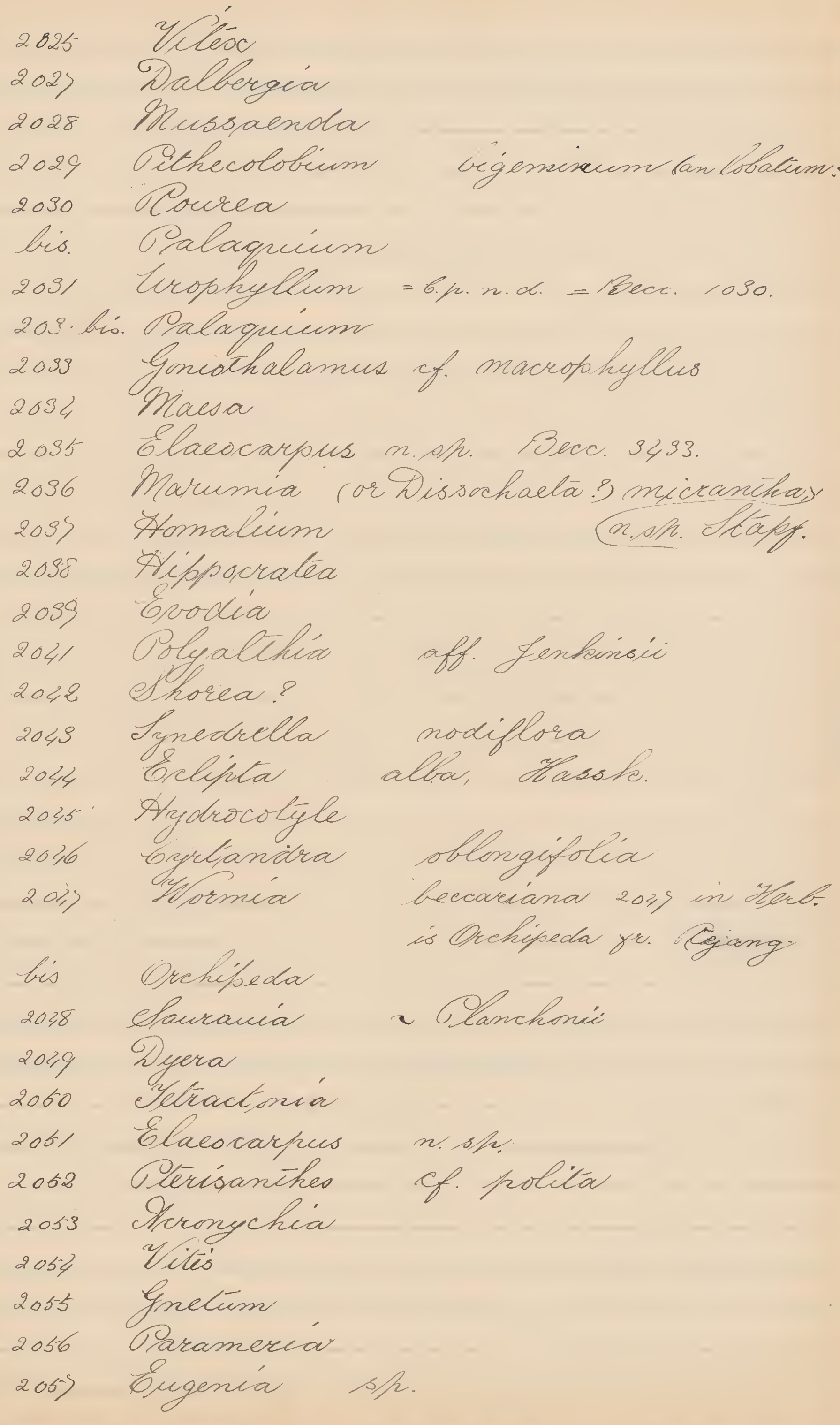




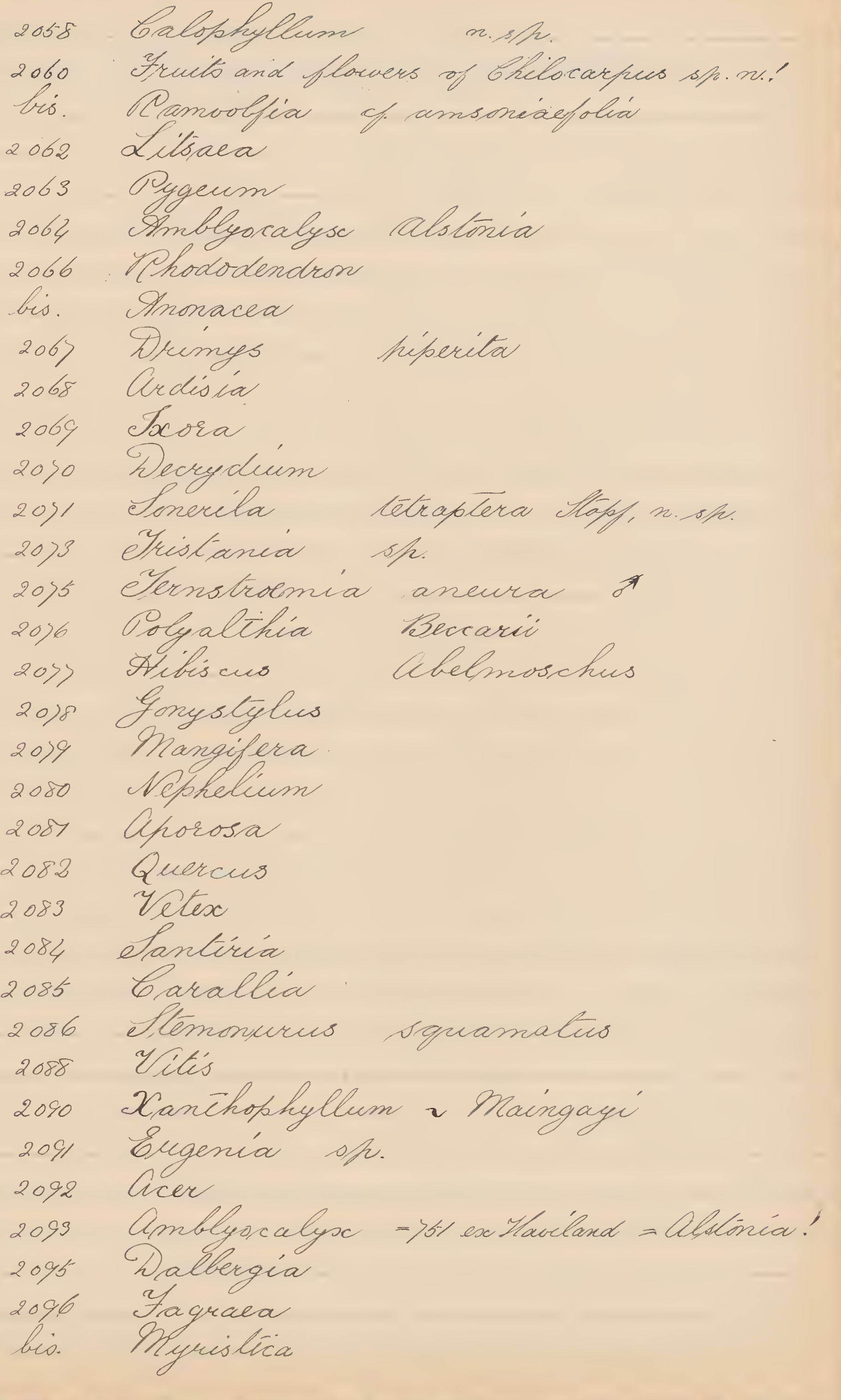




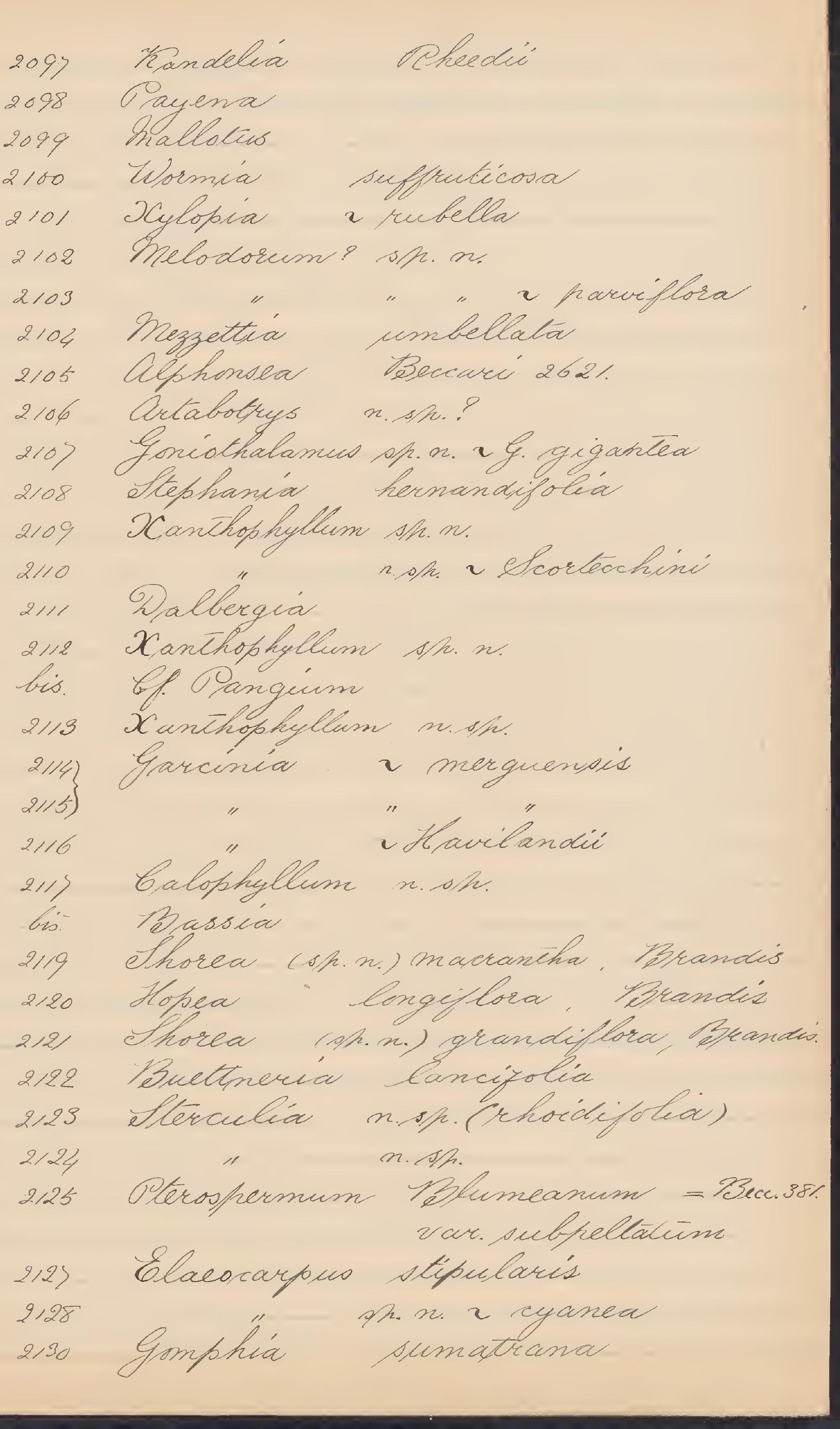




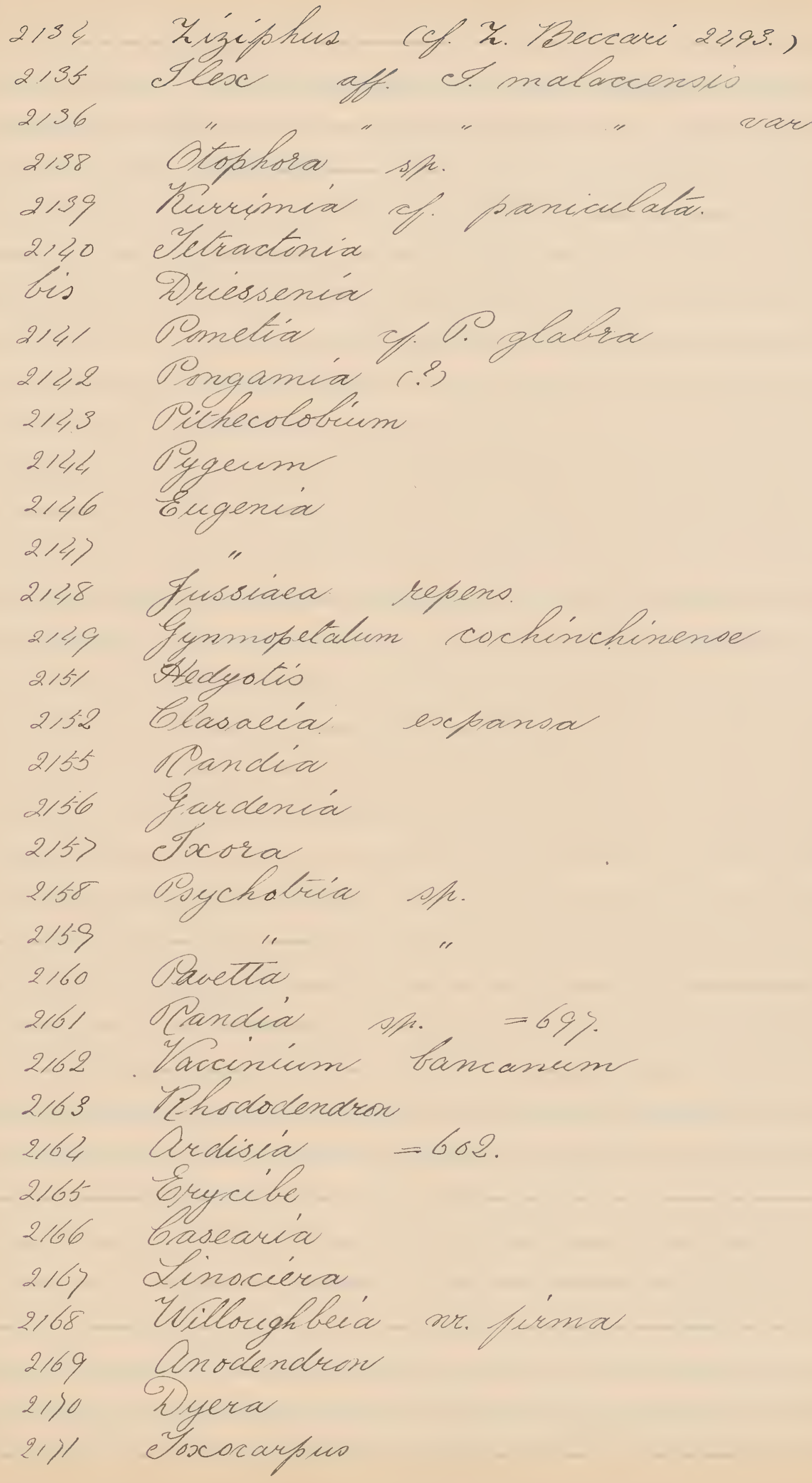




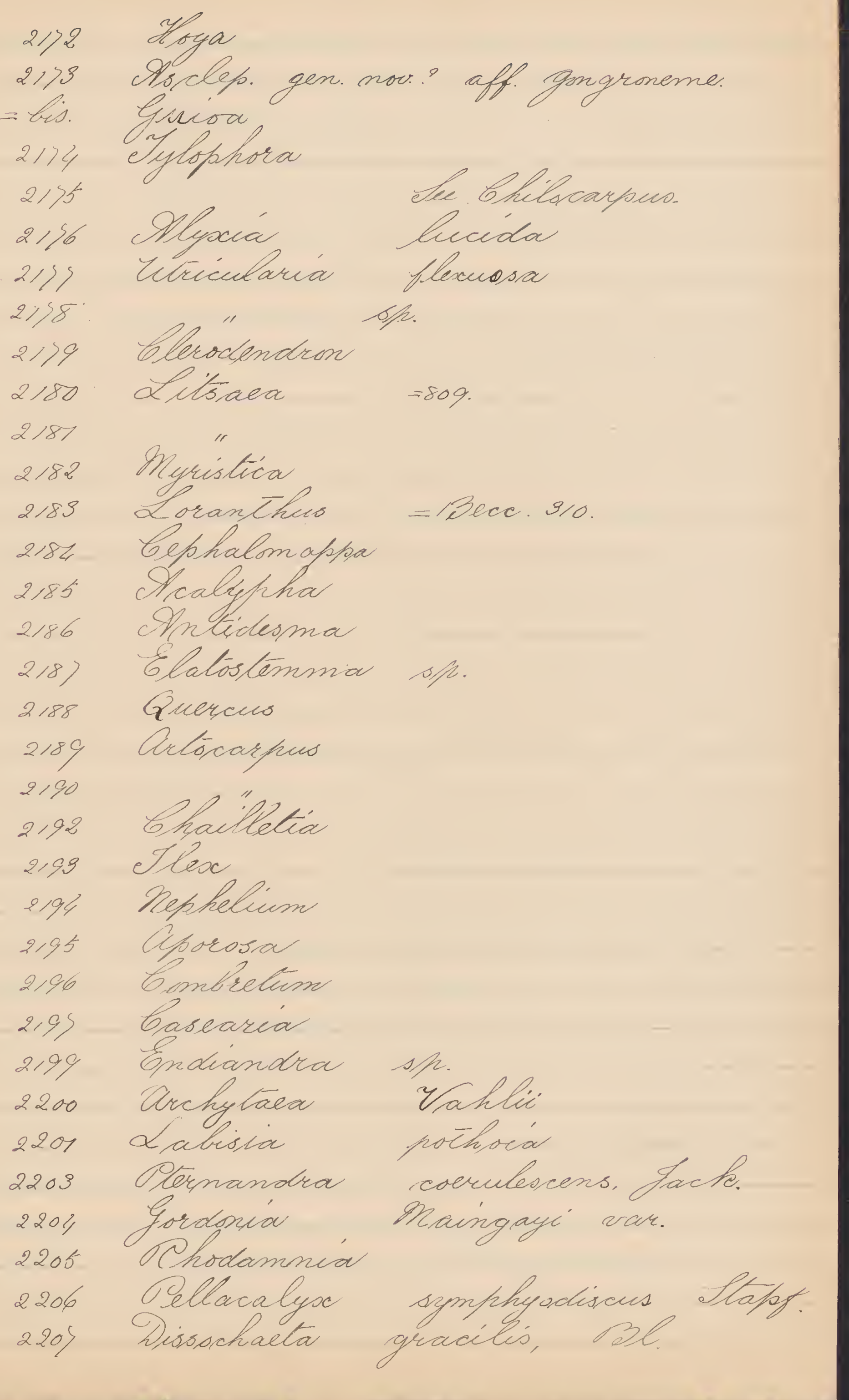




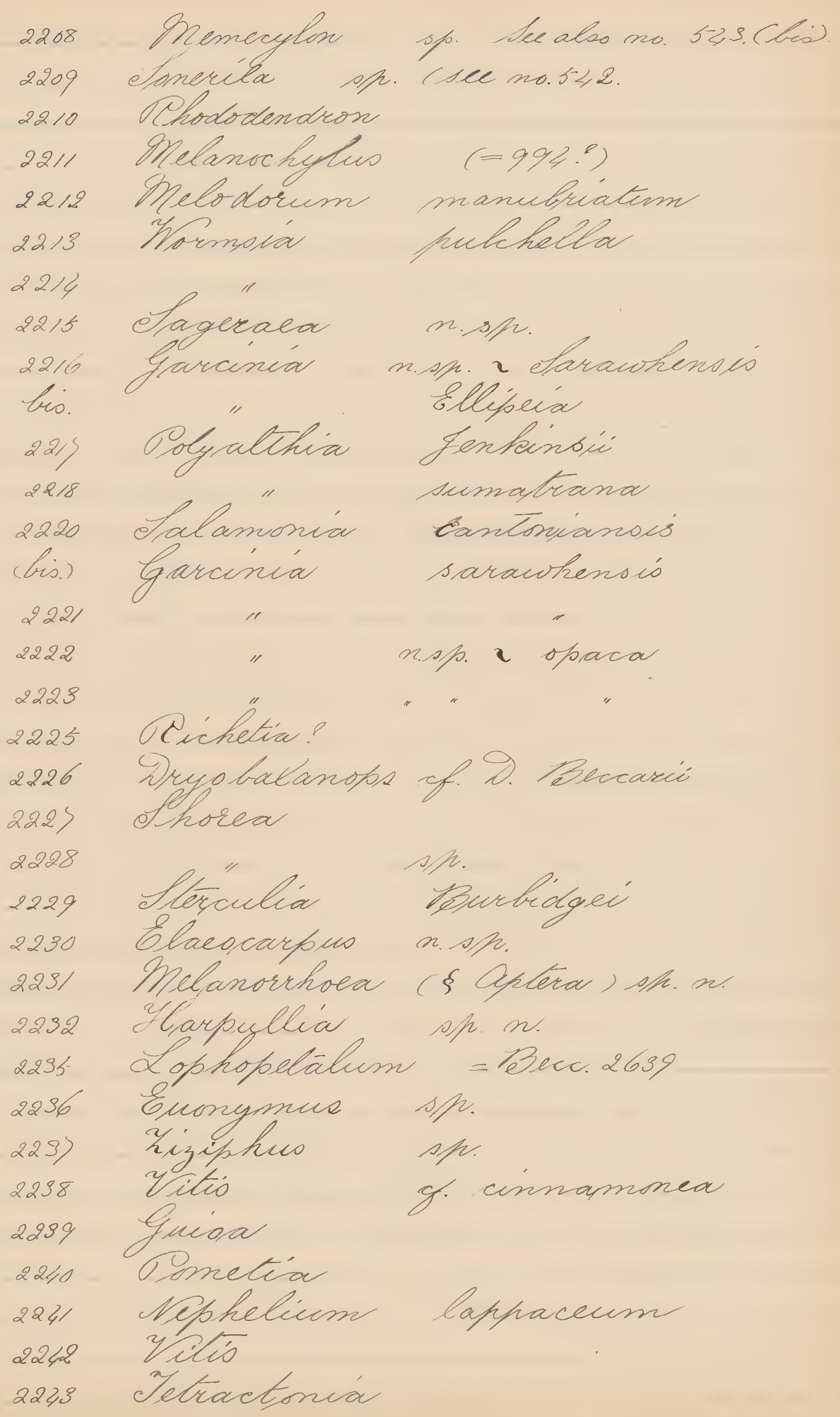




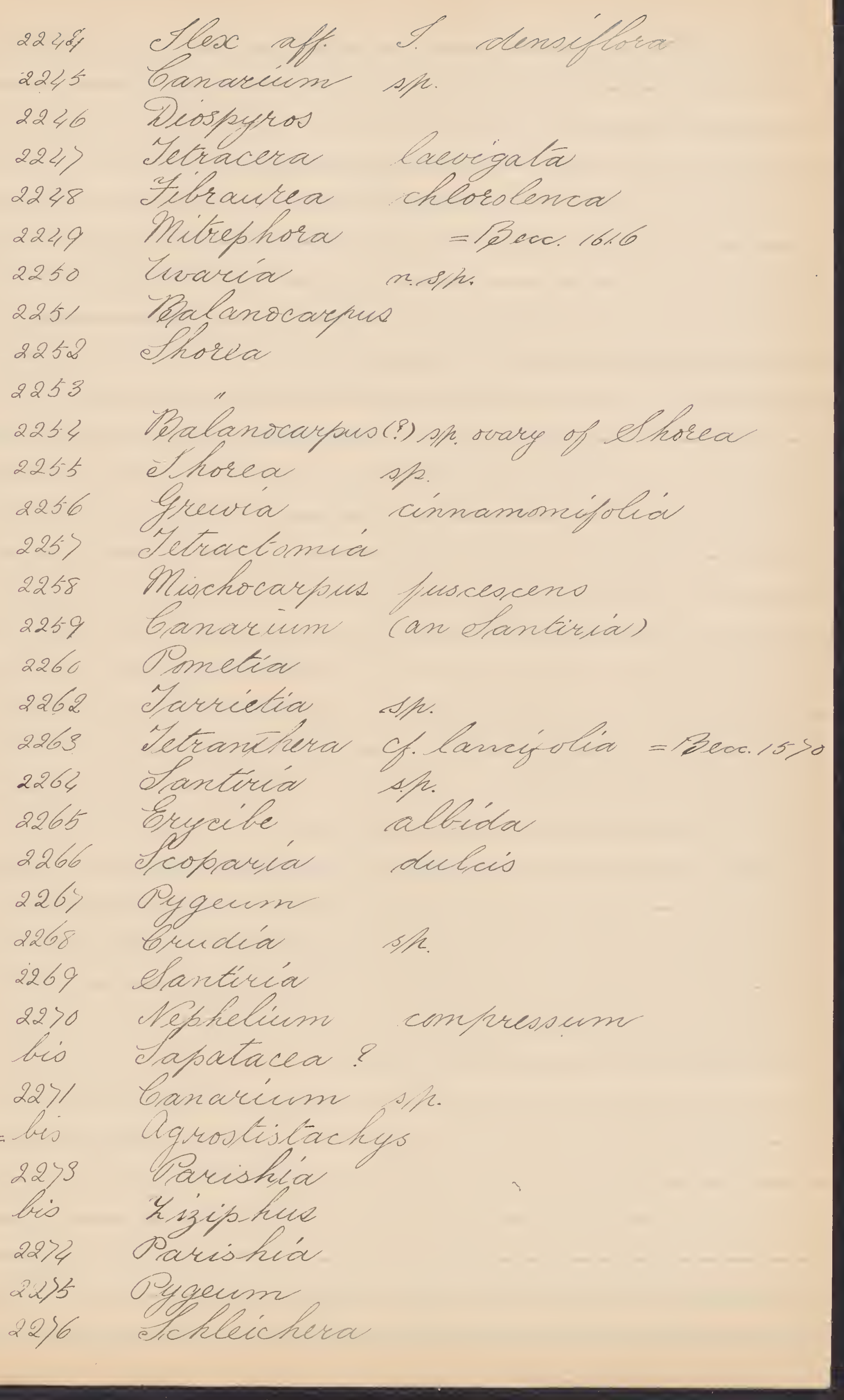




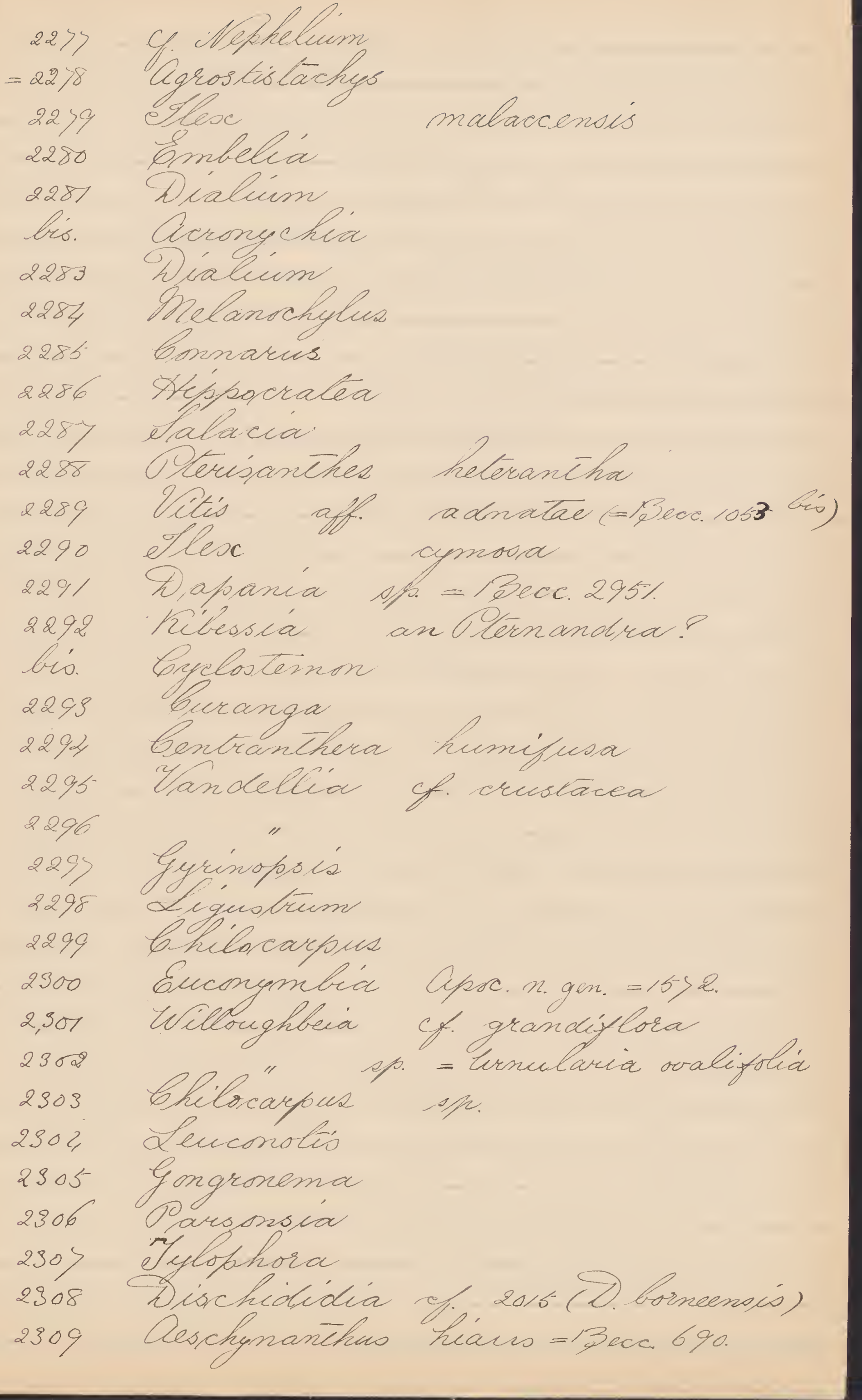




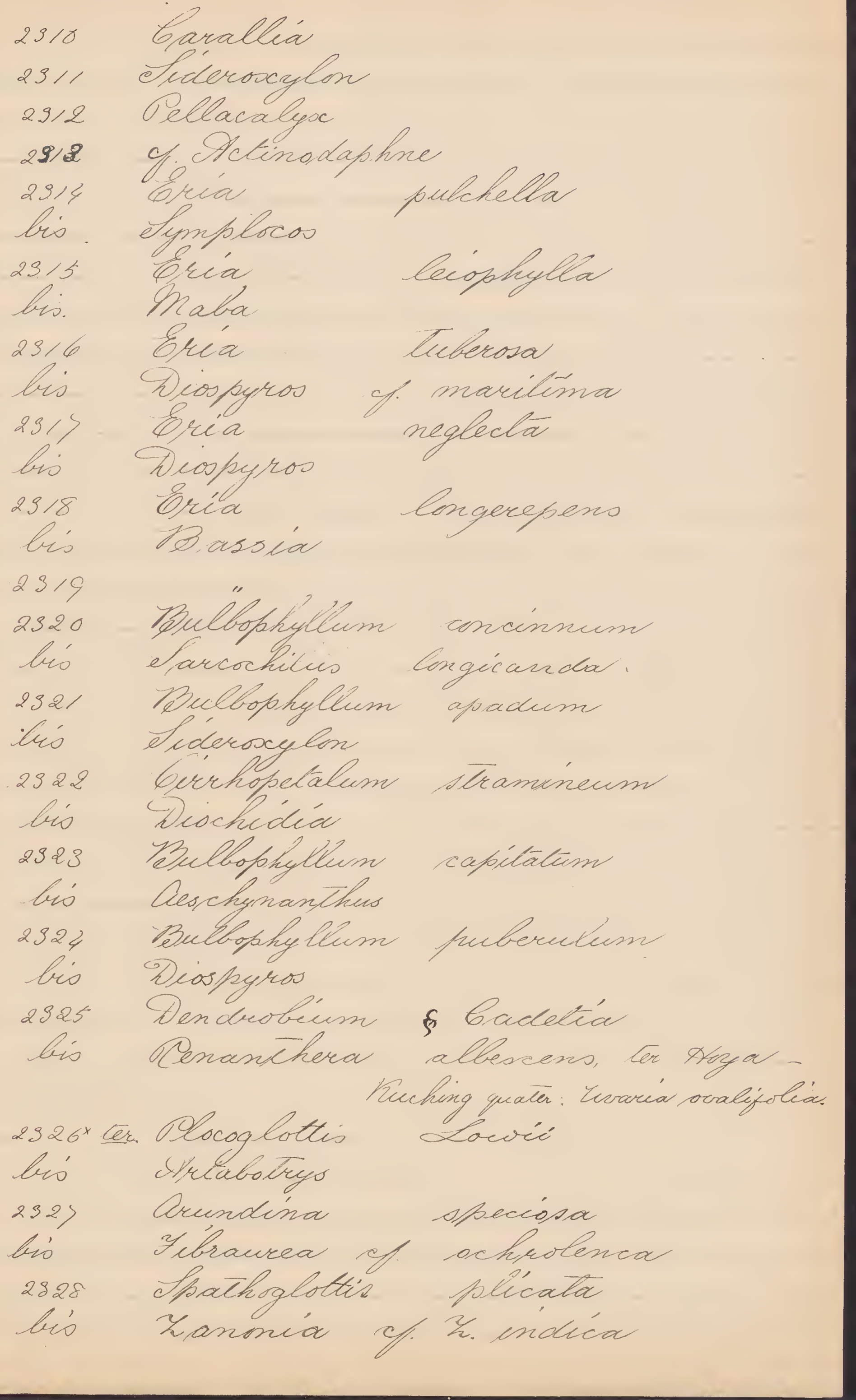


2409 Gelonum of. I. glomeration

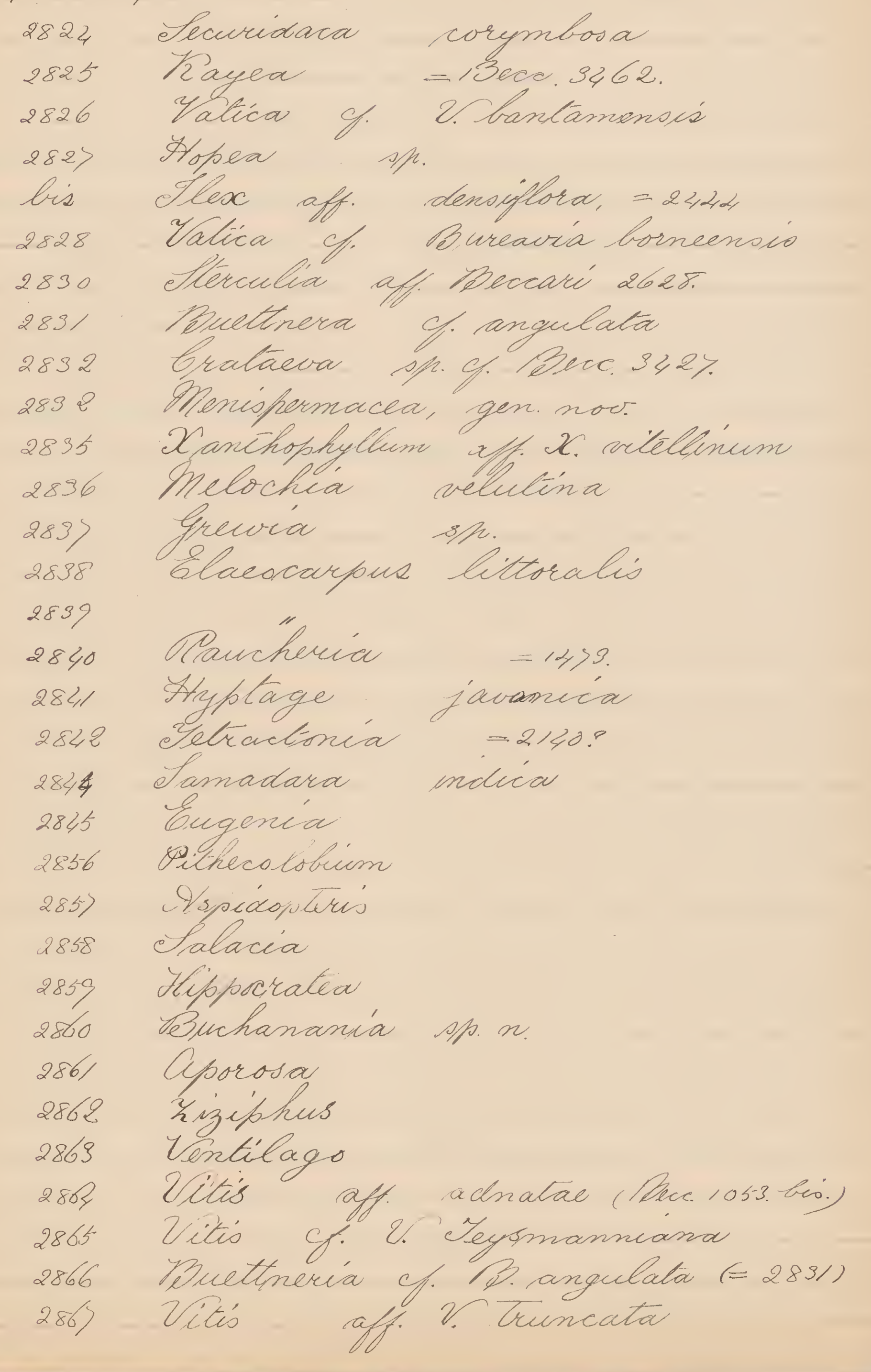




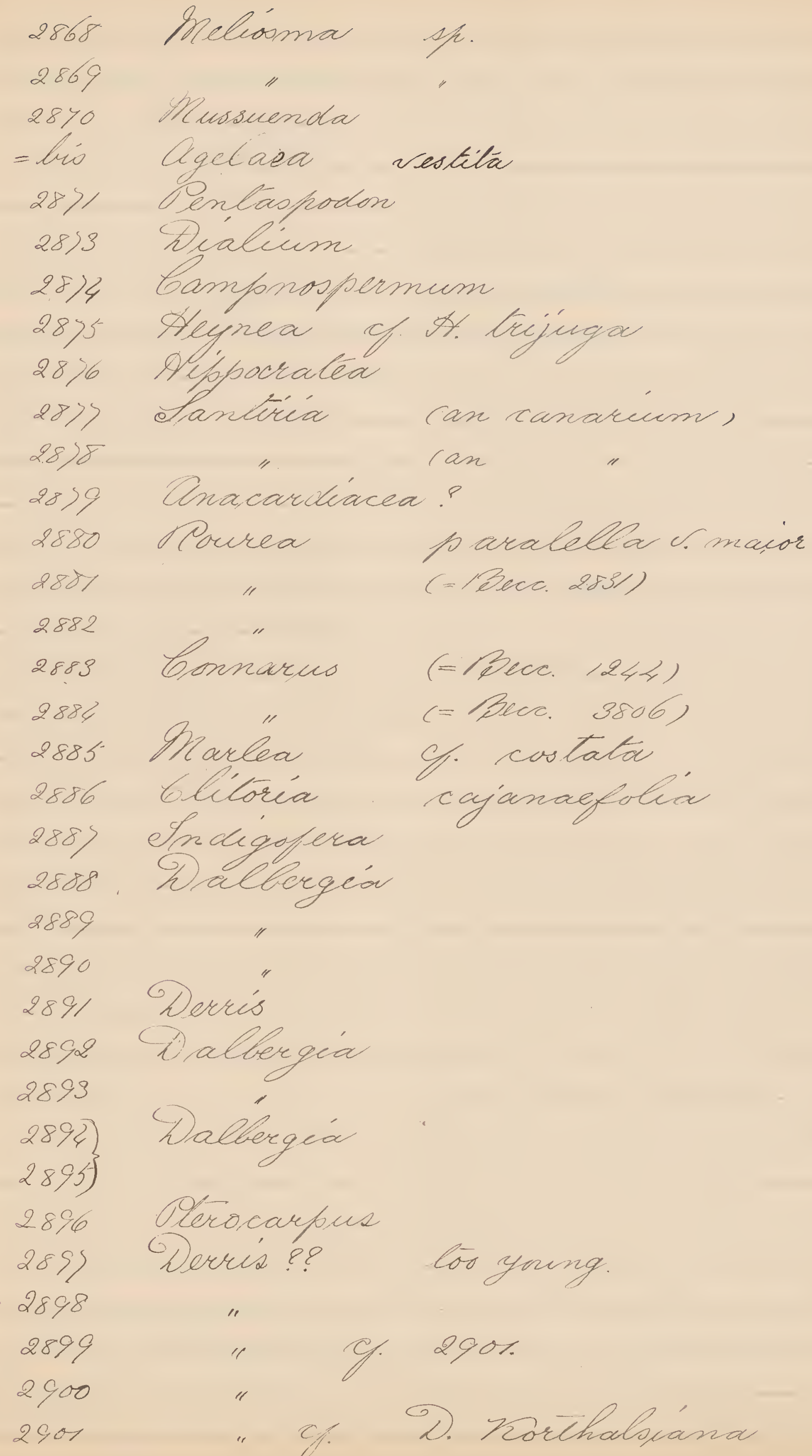




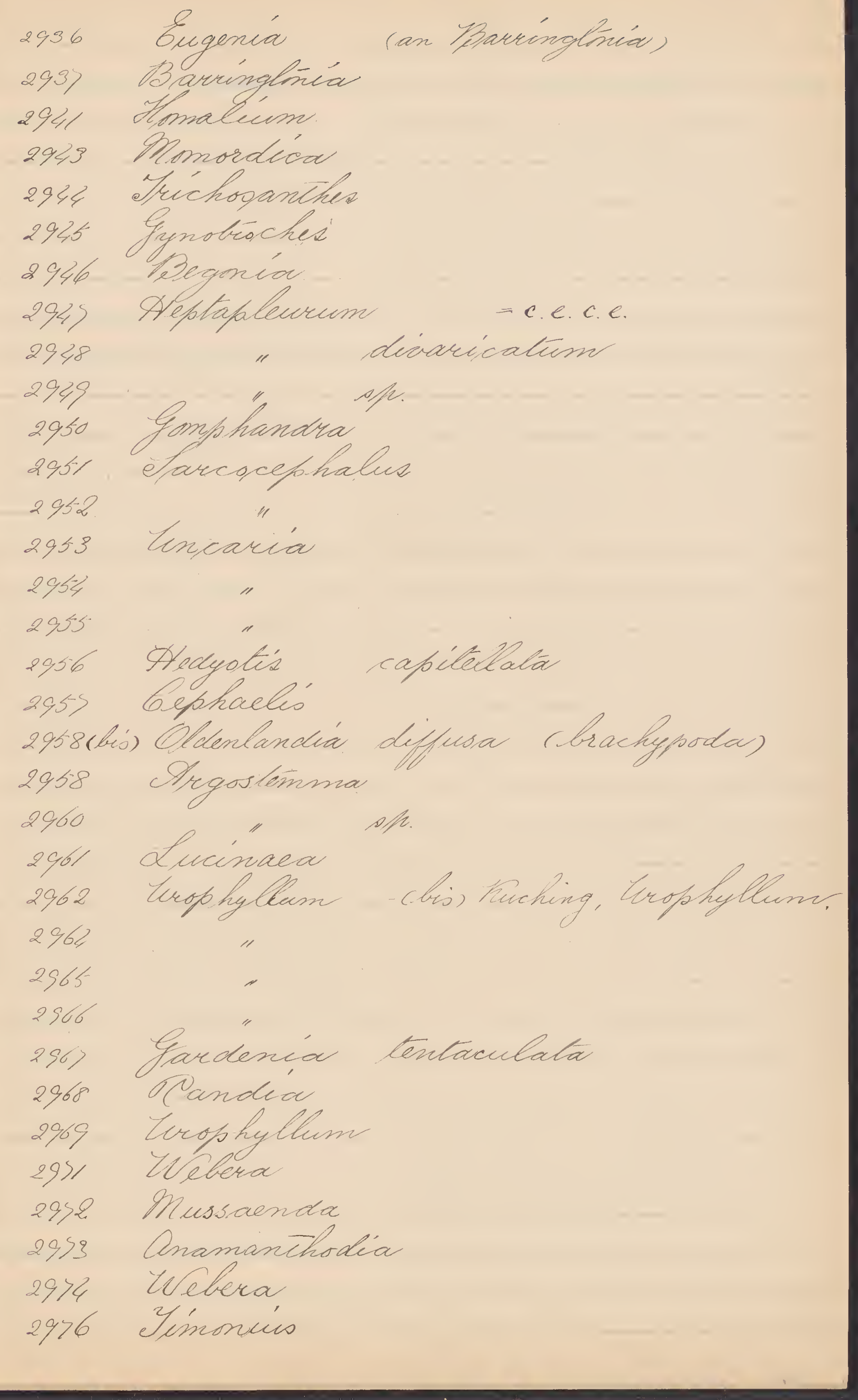


-

297

2939

2980

2981

2988

2983

2984

2984

2986

2989

2988

2989

2990

2991

2998

2993

2994

2995

2996

2995

2998

3000

3001

3008

3009

3004

3005

3006

300y

3008

3009

3010
Goelospermum

clocora

vebera

Griomatomerís

Invenda$$
\text { " }
$$

Laseínthus

turophyllum

osycholiéá

sychodrea

Plorinda

Ssychotría

Gacelinera

Ghasaléa

,

Prienda

bephactis

Ghasale's.

Gephailes

Dyekatriá

Chacaliá

Mryetioluia

Ghasaliá

Lasianetus

Blectironía

$$
\text { Ganihün }
$$

$s / e$.

y. viridéfía

eriédiflora

th.

citríloliá

espansa

C.

(Canthium) 


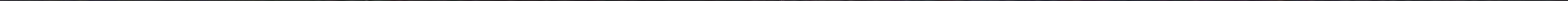




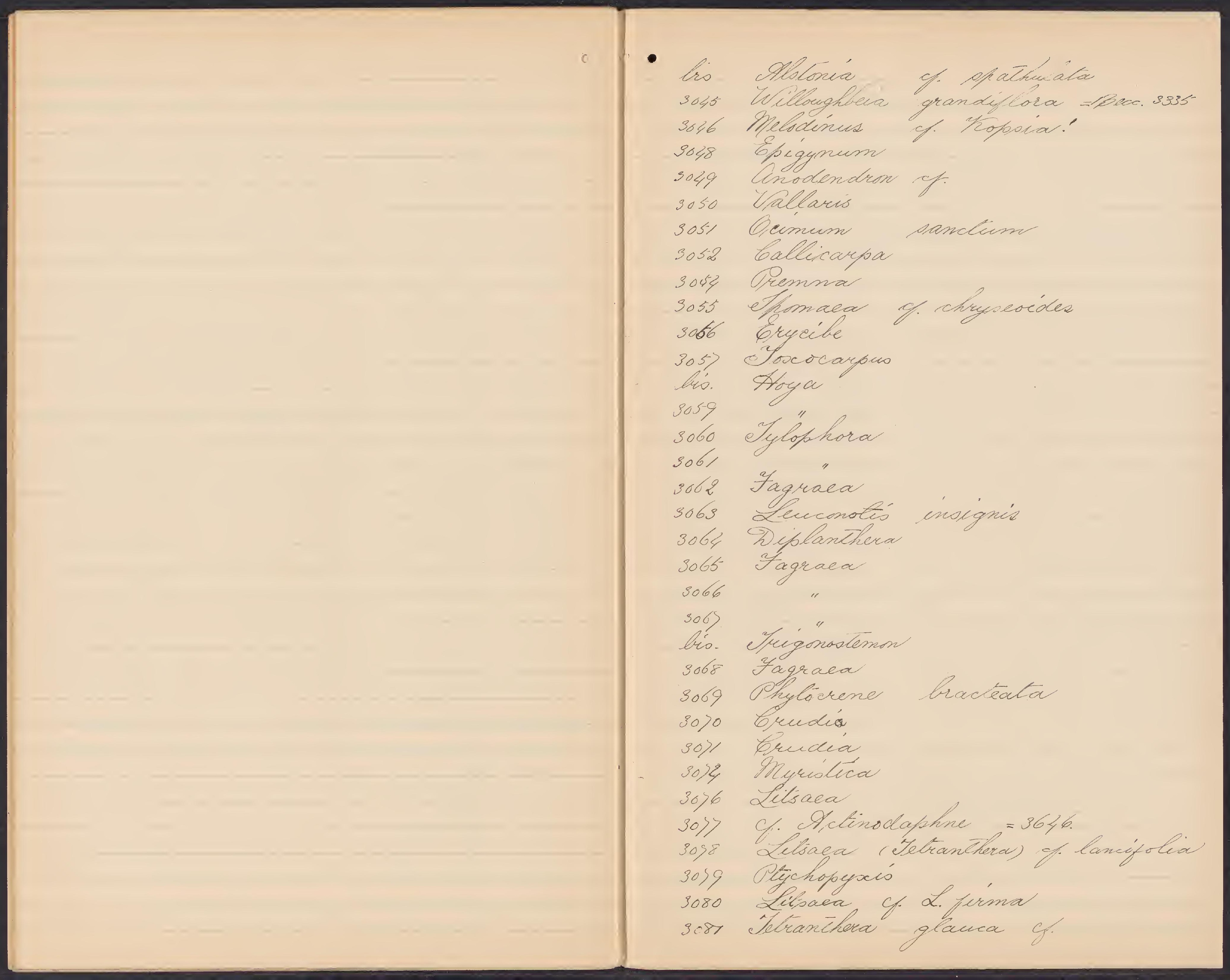




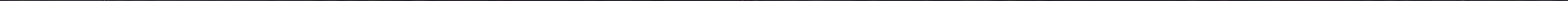


$3 / 26$

3,25

9128

3129

3130

3,31

3\%92

$3 / 34$

$3 / 35$

$3 / 36$

$313)$

3138

3199

3140

beo

s/4t

bis

3142

$3 / 43$

$314 \%$

$3+4$

3148

bis

3129

Erycibe

Pimeleadendion

Alax

Alternanthera chuclase

Prodecca

Clenomeris
Dracaena

Hacalna

of. gracilis

"Anilax

Turbidgea

truff to coryne

oiflos halna

Ireycinetia

Homalonema

Olygonum

Eleusine

Lycopodicum

tourculego

Corpisblastus

Phylócrene

Xylofica

Ohyllagathé

Concrila

Anflectrum

Disschache

indica

ihlegmariá

nummulariaefoleumo

Melanoirhola ¿halansma

Yatíca

Polyalthía

poepherura

sep

- borncensió devaricalüm frallida

(s Aplexw) sfo.n. sfe.

sh. 


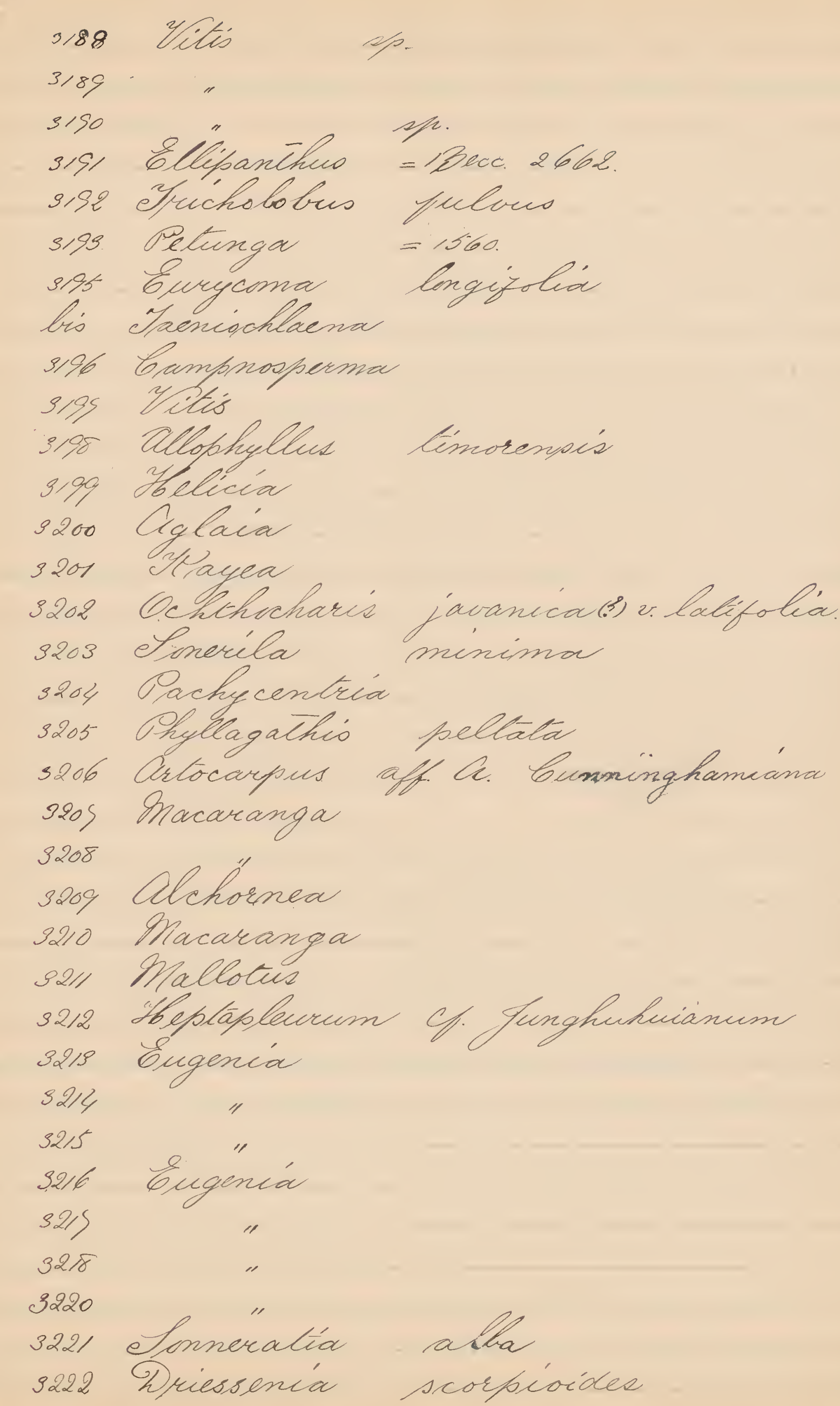




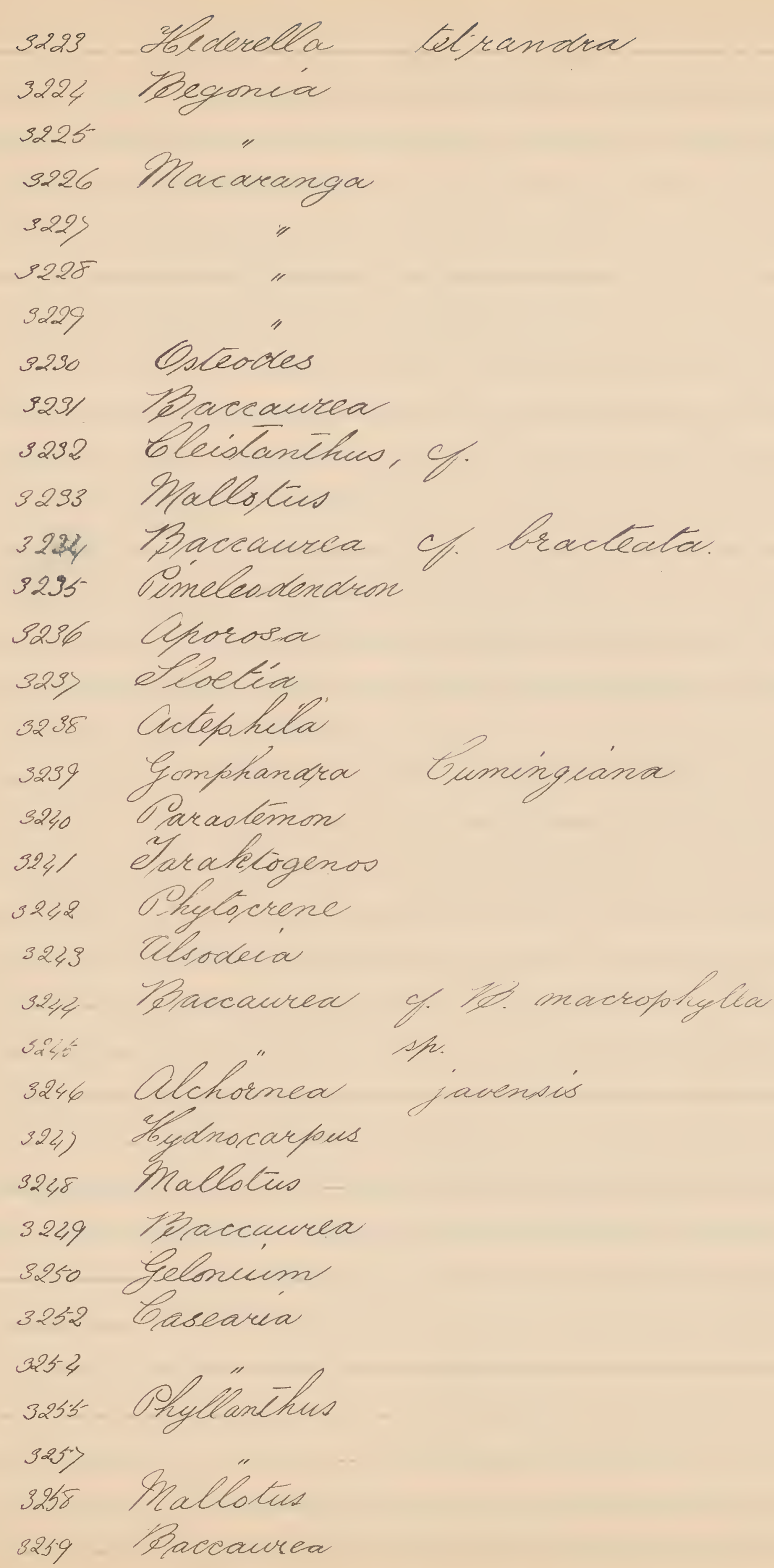


326 Shyllanthus Littoralis

3262 Aporooa

3264 Antidesma

sab5 Aporosa

3266 Lumuigera coccinea

bis. Miglanehus Littoralis

3263

3268

Trucemnia

3269 Aegiceras

32) Whizophoracea

32)2 Fellacalyse

3233 Parkia

3234

Sirecto

ithecolobium

Singulares

32)5

efantiria

ethathalobus

3256

Dalbergia

3277

3299

Millelía

3280 brololaria

mi pseudo-etissoo.

328. Milletía

(c). also Derus ityersiflora)

3282

Desmodicin

3283

3284

Dalbergia

3285

Marlea

3286 Alsodeía

3287

Gomphandra

Oper

$2 /$.

3288

3289

$32 \%$

Byristica yisicum

3292

Oiper $\mathrm{C}$.

3295

oper

crerucosa

3294

3295

9296

Cryptocarya

Cumingiana

muricalum

-

stylosum

sinalim

Ceantamense

ne. Wightiana 
Lisaca

3298

"

3299

3301

bimanat

3302

Prudelia

3503

3304

3305

Pyeistica

('oposfiélater)

3906

3305

3308

3909

" "Yymenacanthera)

conleáalá

3310

331

$33 / 2$

3313

$33 / 4$

3315

3316

$39 / 5$

$33 / 8$

3319

3920

3321

3322

3323

3324

3325

3326

Yiscum

bassyetha

Artorarfues

integrif otea

Elatótémma

Orulyolzia

Sochmería

Sipturues

Ficus

aff. Es malabarica

argentenss

Gastänopis

Quercis

Litsaea

(Gylecodaphre,

3328

$-3299$

3329

3330

3.33

3332

Dehacasia

$-3299$

\% mirocarta 
3333 Eryptón arya \&. densiflora
3333 Qveruia (.9) sp.

Pielodorum sfe aff. Peca. syss.

333) Xylopia sfr. ClBecr. 3335:

3358 Foniothalamue

3939 Qulabolrys

3340

3341

Solyallhía

3322

$33 \% 3$ ballophyllicm

aff. Deumaliana

3344

3345

3346

3345

$33 \geqslant 8$

Y.

Garciní

Diospyros

spe.

3399 ethorea

3951

Anisobhyllea

Majlopia

3352

Yibranrea

3353

spe.

3354

Yarcínía

3355 Tallophyllum

3356

f ossepsicion

935.)

eqlesc

$=1663$

3358

Tetractomía

. ph. (Bece. 1908)

3359

3360

"

Bussaendopsic

rhlorolenca

336, etalaciá

9362 eluintoñá

3363 itandoricum

3364 itantiría

3365

Inaclletia

3366

Oheus

3368

Banquilexa

C. barbadense malaccensis

$s / \mathrm{s}$.

sh.

- becarána

sfe.

Cf. glavea

marqinalúm

el. et glabrifolex

sh.

sh. 
3369 Dischocarpus fuscescens

3350 ifalacia

33), Pleléacea

3952 Nephelium

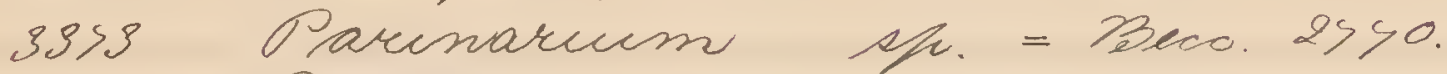

9934 Dablergia

9355 gmill"

3936 Dilletia

393 Baphea

3378 Thomalium

33)9 Thusphora mucronata

3380. Exyenia

$338 /\}$

9.983

Sconanited

3 e.

3384

3385

3386

3387

9388

3389

lis

3391

3392

3393

3394

3396

3395

3398

3399

32,00

34,01

3402

3203

Eugenia

Dissochaela

Predinella

Ibederella

Pachycentra

Memecylon

theficaplewum

Viburnum

Mussaendopsis Deccariána

Bedyotis

Xianchopingla

gracilis

lorata

tuberculata

caloneuron sp.

$=$ Becc. 231 .

- "fruticulosum

Grvodiá

veridis

Elaescarpue

Pentace

spe. 


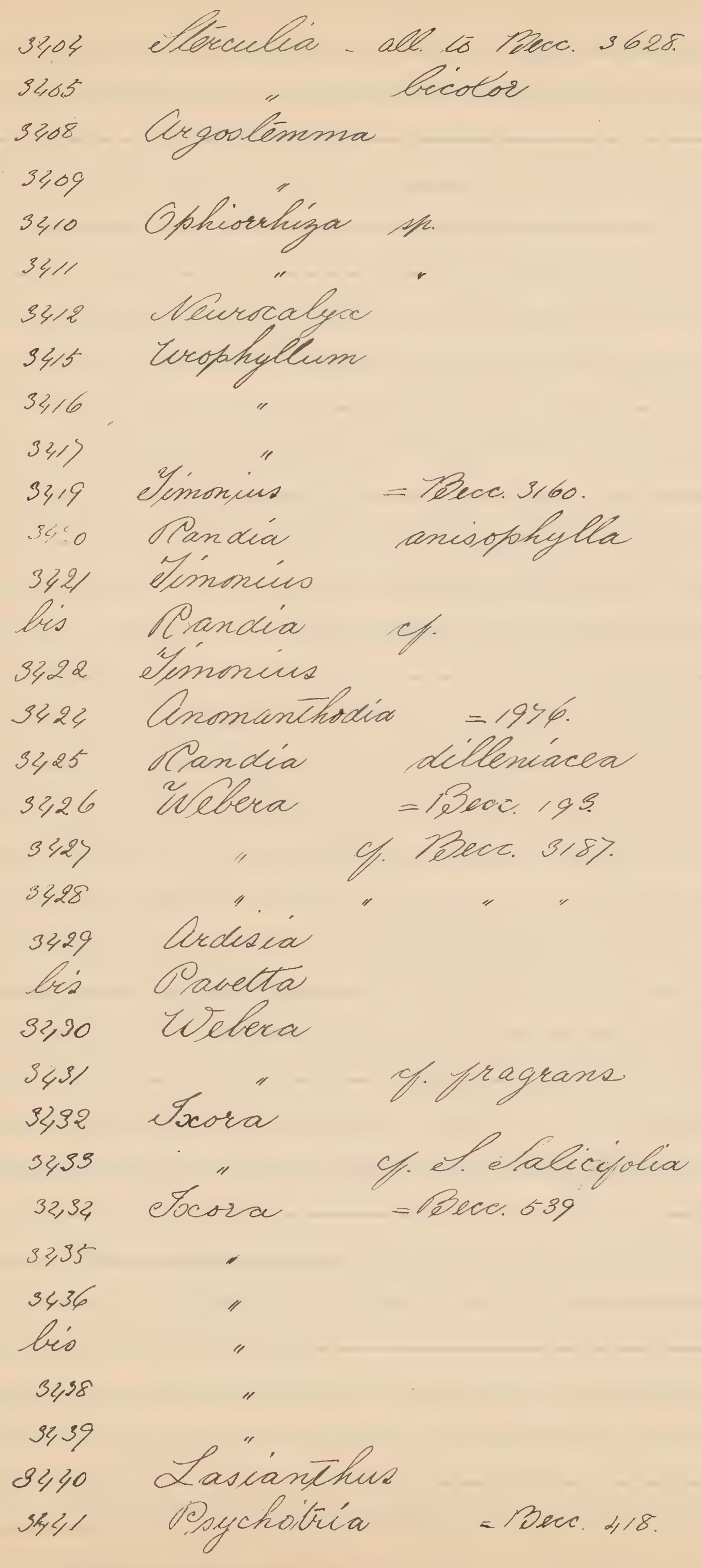


12,2 Taedería

342,3 -18ecc. $125 \%$

sil4 Gardeniópsia = 304

3245 ifrisciadia

346 Ganthuim =13.2993.

sily) "see $3008-3009,300$.

3iys Ebydnophytum

3451 Yacutnera

34,52

3955

3254

Syychatica Chasaliá

34,55

34,56

lis

3458

32,59

3461

Psychotría

- Bece. 3618

"

- Thece sors.

Jocora"

Oneviá

Mykconecuron

escora

3462

Dychatría

3468

Siblycosia

32,65

Cigislepie

3466

3465

32,68

3369

bis

32, 70

34, 1

32,58

$34>3$

3434

34,5

"

Maesa

Embeléa

Macsa

Yacelinera

-666 .

sp.

Maesa

3276

34यy

3498

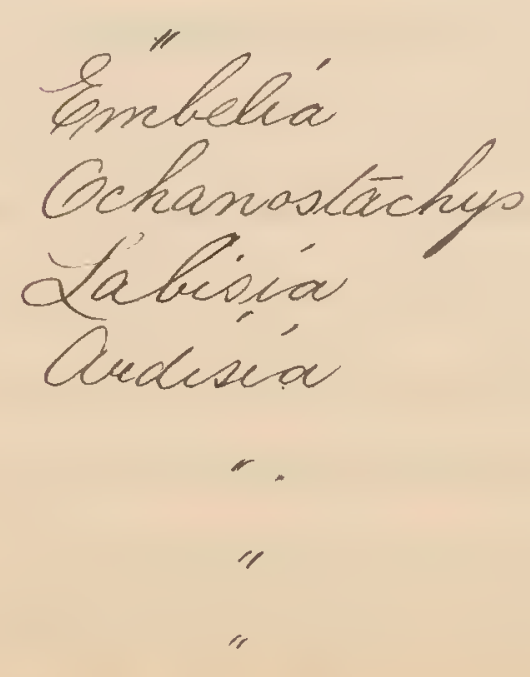


Tymplacas

Massía

3481

5482

3483

3484

C: Dísfuyras

Garcinia

3485 Salaquium

3486 elvocarpus

3isy Willoughbeia

3488 Linociera

3489 fasminum

3190 Chelocarpus

3291 " " decipiens

34,92

32,93

Willoughbeia

eloxicarepou

3294

trapminum

edulis

34,95 Dyera

3396 Chiloparpus

3495

$3 i 98$

turceola

Anodendron

32,99

fagraca

9500

3501

" "

Casearea

bis Yabernae montana

9502

eylophora

3503

3504

Asclep. Gex.

3505

9506

$350>$

Tagroea

3508

Bibrosacme

3509

Jorencic

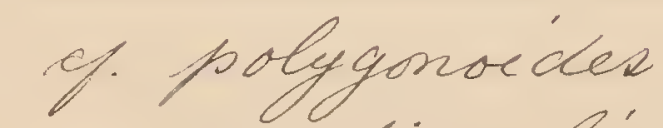

andella

Gentrantherd

cf. urticifalía

3511 Donnaya

3513 Hoygrofhila

hemifisa

replans

salecifoliá 


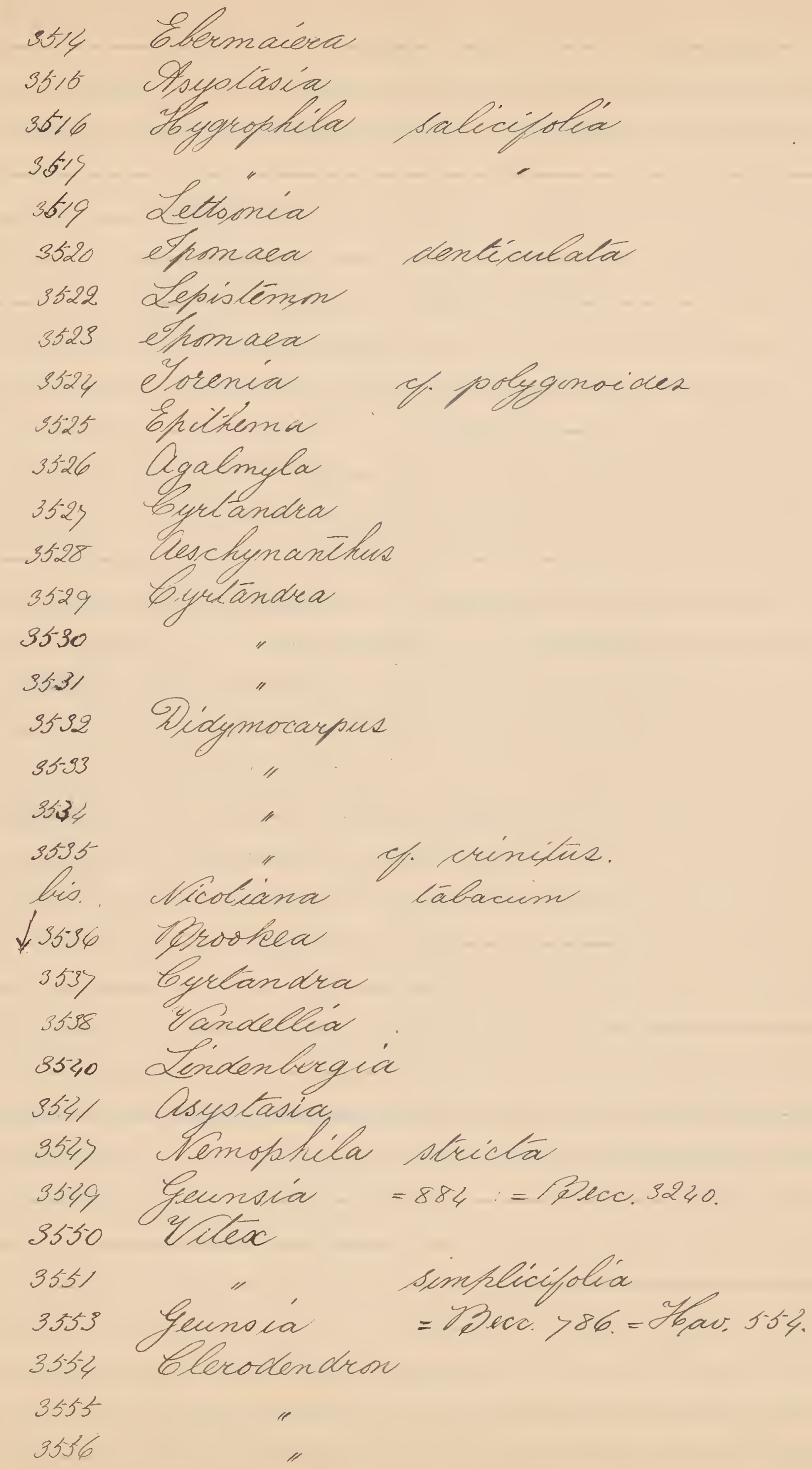




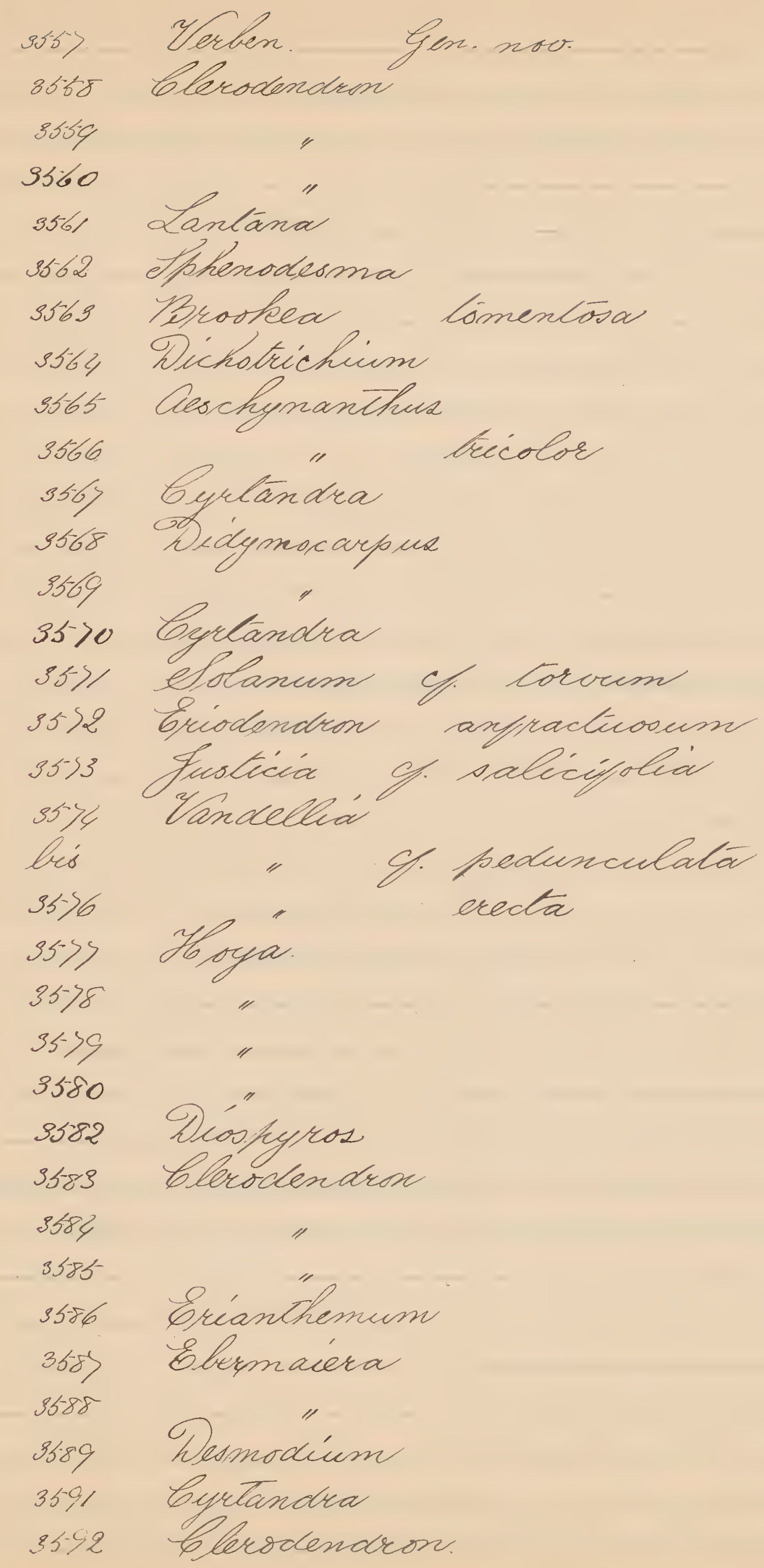




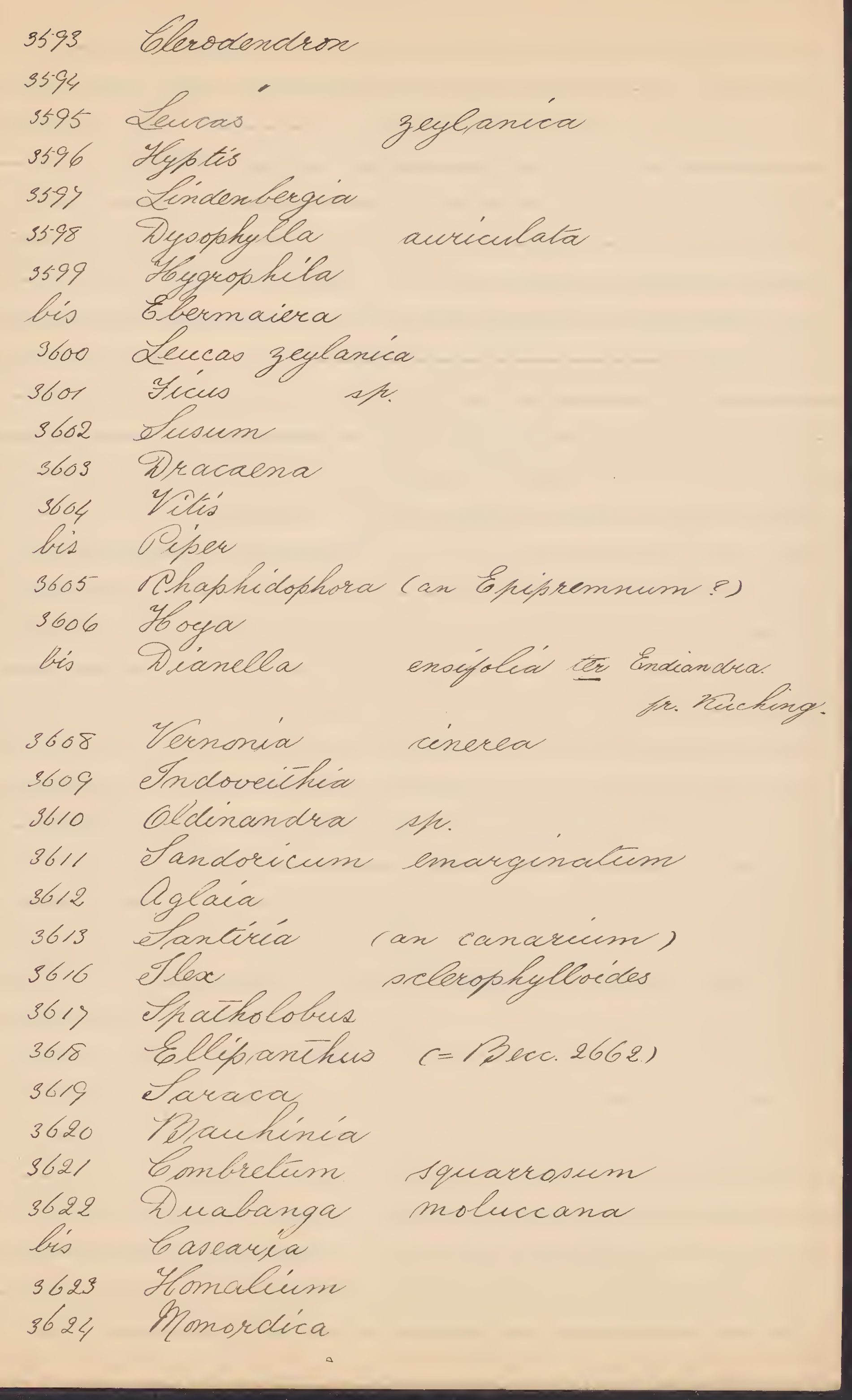




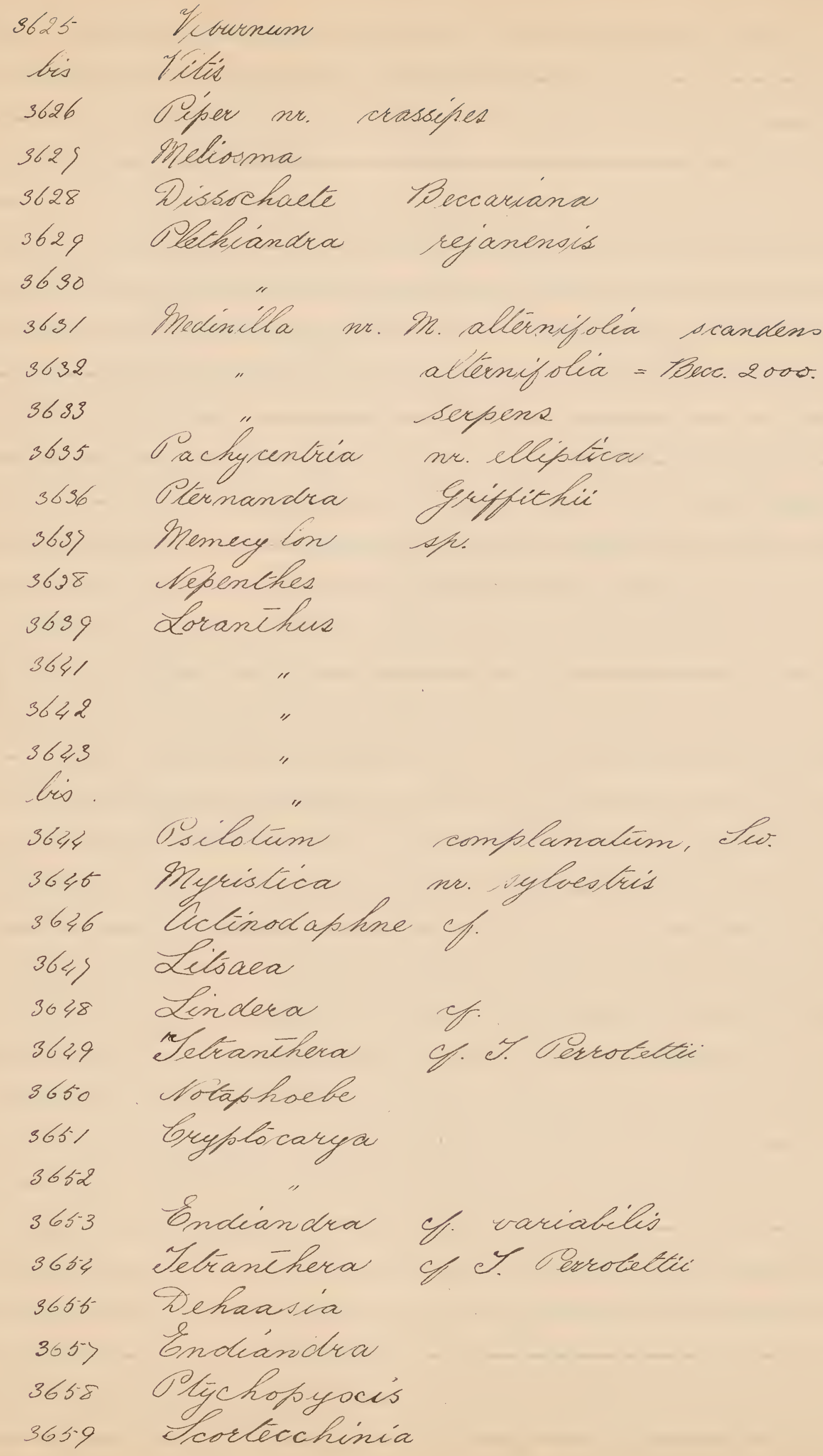


,

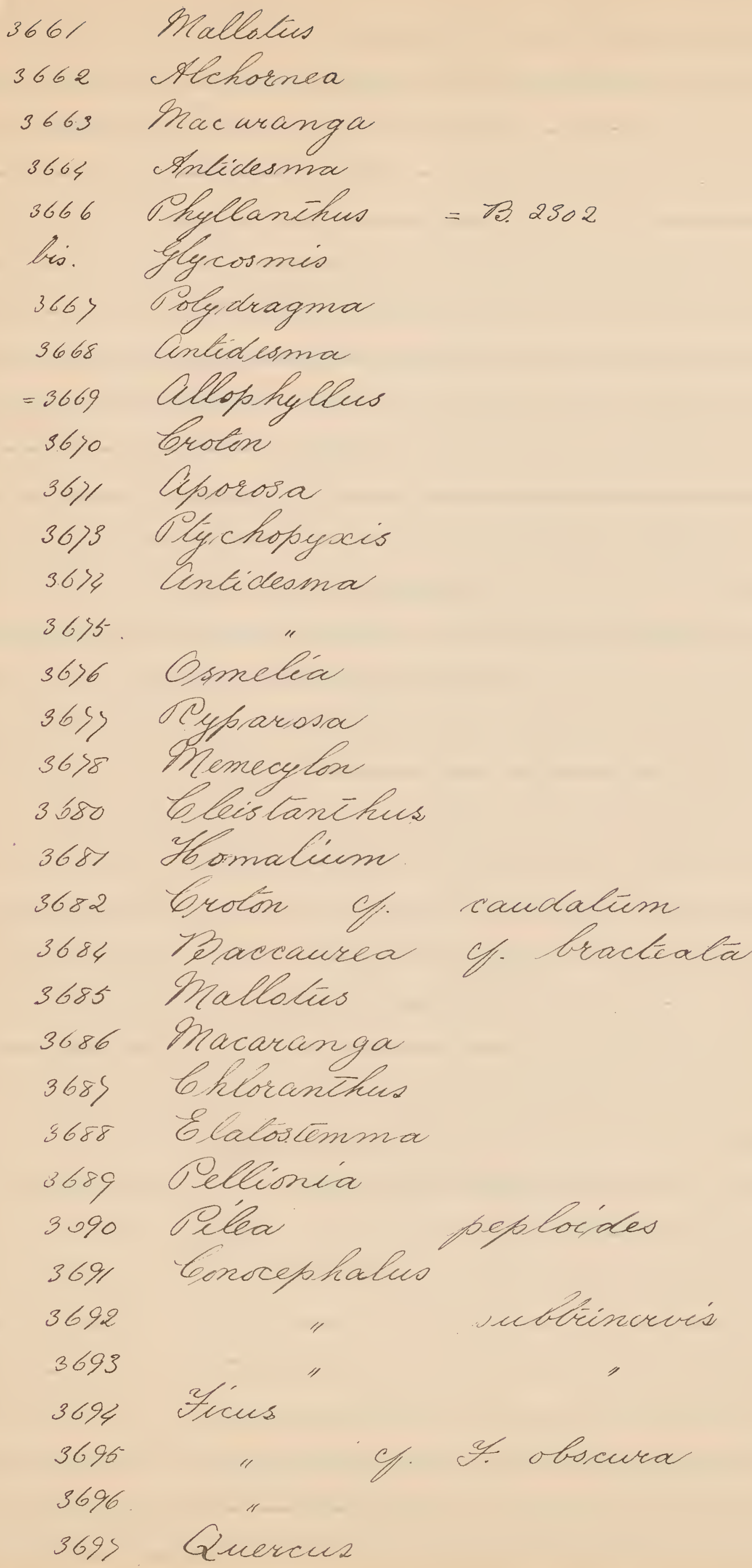

caudalum

ff bractéata 
bis Tecuo

3698 Grastanopeís

3>0 Quercus

3502 Dacrydium

3)os buidia

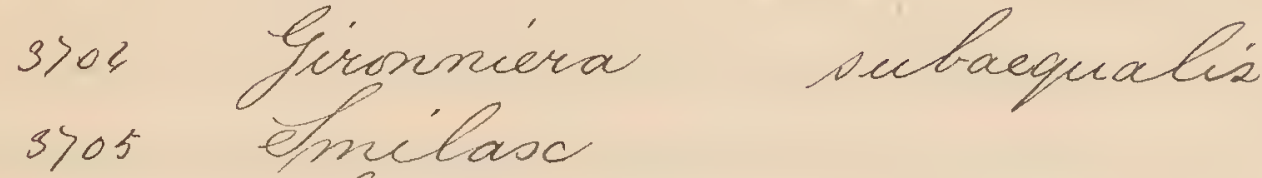

3906 etymplocos

3y os Reparosa

3) 08 Flacoutía

bis Erycibe

3)09 Tebastiana thamaclea

3) 12 Malanocarpus.

3) 13 Eufthorbia filutifera

3)14 Foniócaryum

3)s Aralidicim

$3 y 6$ Loranthus

$3>13$

\% lex

C. Sipndenianus

3) 18

Dinar hloa

3) 19

Giganihochloa?

3) 20

Dinochloa

372, Osmielea

3)22 Thyllanthus

3) 23

Gironncera

Lepisanthes

$3>24$

9925

3) 26

Erycibe $\%$

Orycibe

Byristica

3y2) Glycosmís

3) 29 Ceyfurosar

3)30 Arolisiá

bis êtrigoroslemon

3)31 Daclylocladus borncensis

3)32 Doflelotus

bio

Aspidopteris

albida

elliptica

$\rho /$ 


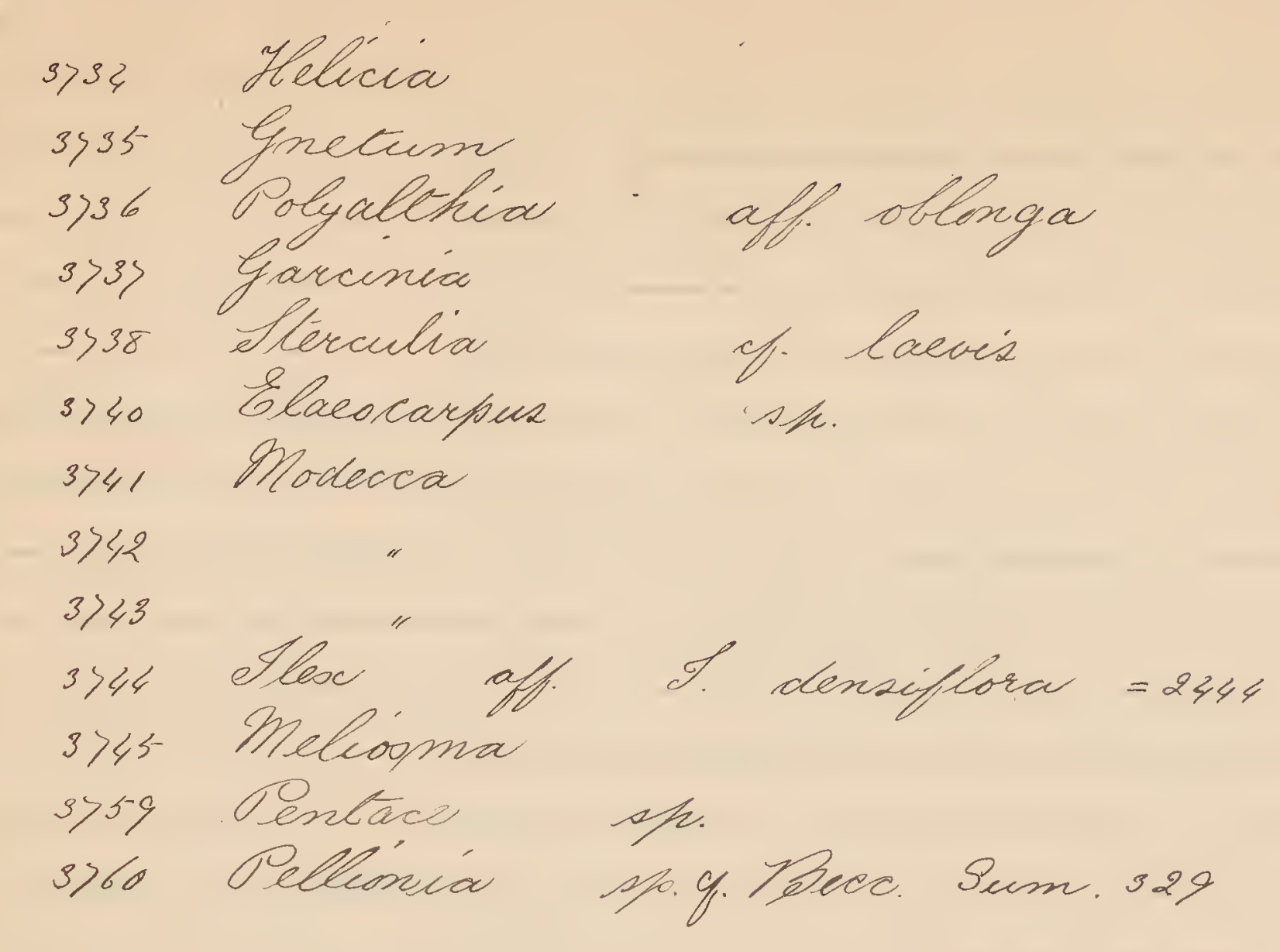




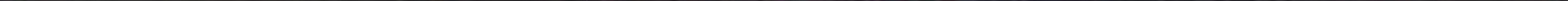


Eallophyllum

Yomphandra Gonithalamus.?

155 Daprania Artäbotrys

160 Bachenía

wr. suavedens Yl.

16 Eccosia alatá

164

165

1) 0

1)

iss

180

183

185

$-91$

198

205

211

$2 / 3$

214

2,5

224

$235^{2}$

241

213

245

248

254

25)

258

Uzpometra

risiphus bianarium

authemis

twaria

Ganarium

Gluta

Aqelaea

Dióclea

ramiflora 86 .

r. Storafieldii, Prig

Yeccarie. Engl.

robecsta, zootefil.

ovalifolia, tal.

Oocymitría

Leea

pteris, anthes Disefsalim Tlemingía etaraca

elernatromía

Ho oopea

Luvenga

etomecarpocos

Derrís $\mathscr{C}$. D.

Bauhincóa

Yaphia

261

ballophyllum

Mylopica.

restita

$=529$. $?$

braunoniana, b.s. Glarbe polita, Ppig. cl. anomalum $3 y$ congesta, Moxls

aff. e? coricicear. tekeff $=30 \%$.

262 Granaruem 


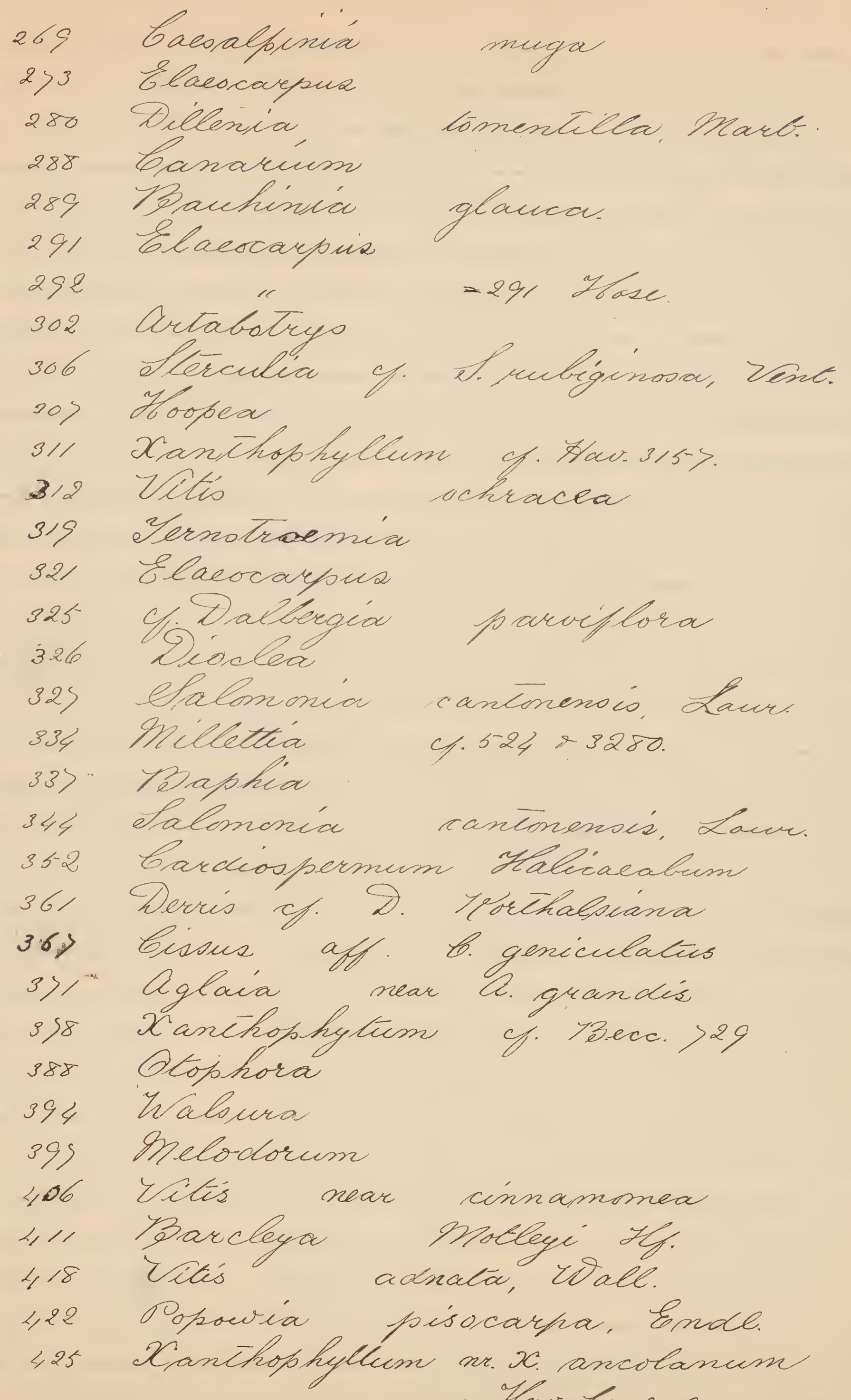




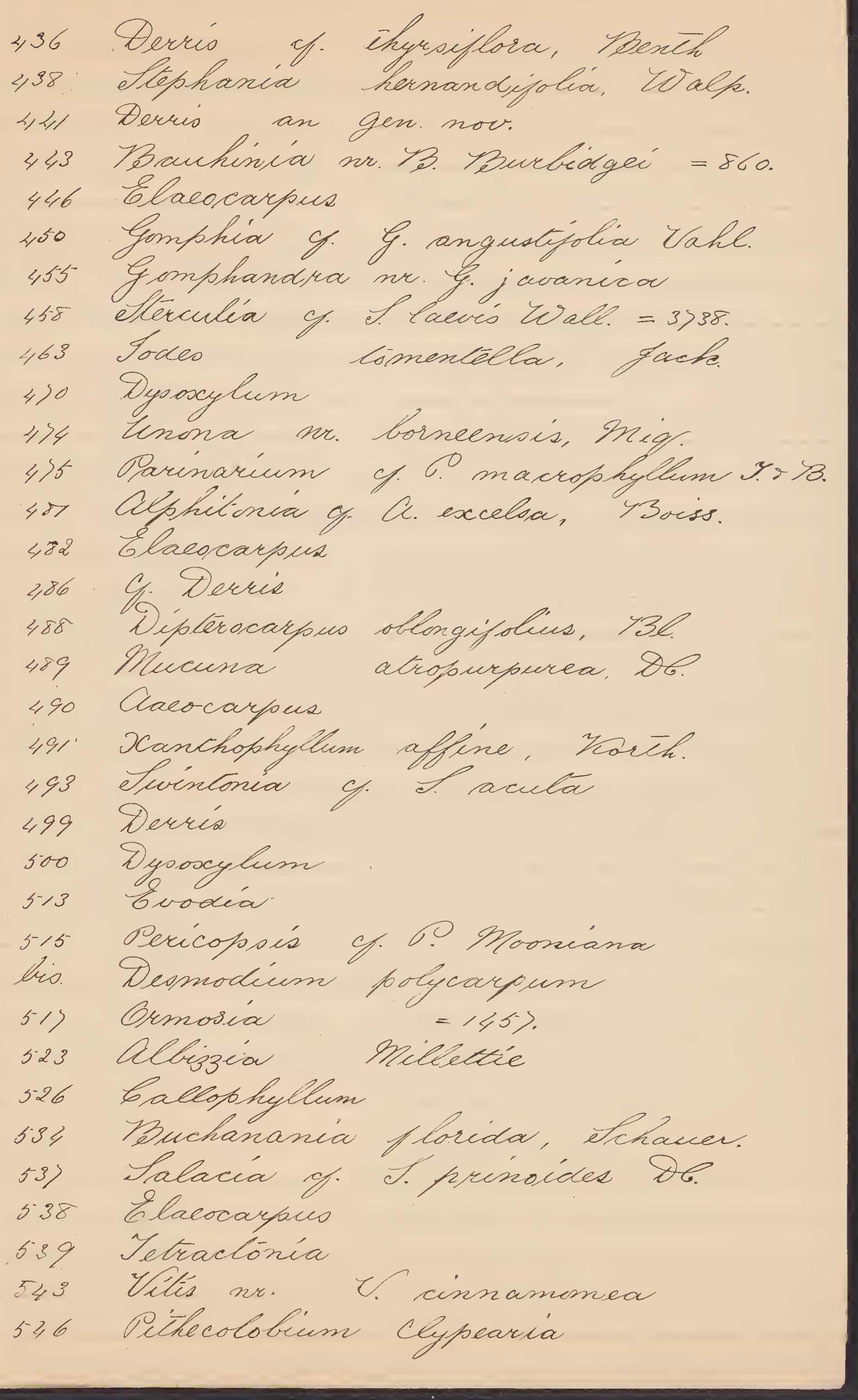


Cravrayí Guiva Heibiscus of.

562

568 .

569

$5>0$

$5) 4$

$5>5$

$5>8$

587

$5-83$

586

590 Pellophorum Guioa \& Xanchophytum Eurycoma Stérculia

Bauchíniá Dalbergía. Dongamía yoo Ibopea Barclega OCerospermum ten

Cf. $x$. cordalum Bl.

odoratisima, Y3l.

Beccarie tsing

G. plewroptêris. Madll.

teleaceres, \&.

ferrugineum

- plevepterí, Padlf. gefolea. Jack.

ferruginea, Posab

glabra, cf.

Bpotleyi y.

5

6

Slimeanum

csubfreltation.

607

605

prycoma

turaréa

maceof hylla

var.

Canavaléa

glabiala yg = valifoliá

611

\%. Derrí

Guiva

$6>2$

vitis

oblusifolia $\$ 6$.

6,3 Clérculia

622 Yoschía

625

Buchanamía

eleǵinosa

626

Grevía

628

fararoríc

G. pleuroptexis. Ipadle.

629

Candoriaum

cinnapmomea

632 Ylaloura

643

Derris $\mathrm{C}$.

grandiftora Mast:

insignes. Bl.

652

Yaloura

nr. If. borneense

654

Adenanthera Iemecarpus

pavonina velutinus, Ying. 

Tuchanania Pithe colobium

$6>4$ Eronymus

6)> etanciria

$6>8$ Dalbergiá

680

Duria c/.

687

685 eléculia Pellophorum Albizizia

691 Mylopia Gallophyllum riziphes Elacocarpus Glacocarpus Gomphandra Laptonyehia elacranja

Is' Elaeocarpus

762 Alphiloniá

>69 Dalberqia

$>>0$

$>>$

$>>>$

$>80$

38

383

384

388

790 Clophora Allophyllus Heleitiera

Deamocium Dyesosegleme of Sylaceum Desmodium trena elida

800 Flemingia

$8>2$ Teravia

Melochía

\section{D}

Bhampioni This y

2. Lesiudinaricum

e. Jerruginea $3 l$.

ferrecgineum

Milletií

$=73$ ecc. 3368 .

$=73 e 00.1985$.

Horopieldii, Mnig

nr. G. javanica

glabra, Terez. var.

C. A. excelsa, y3aiss.

Làmariñcifoliá, Maxb.

lamentosa L.

unbellatum, DG.

arborescens, Plig.

bracteasum

gangelícum

Lobala, L.

opinosar, $L$.

strobilijera

lagopoides, 86 .

velutína 